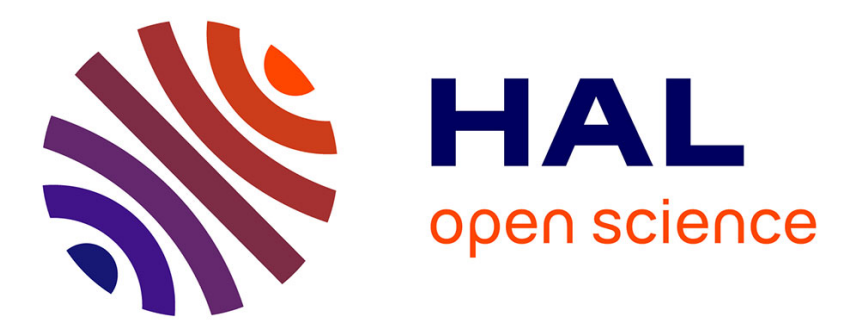

\title{
Introduction aux formes et raisons de la diversité rom roumaine
}

\author{
Martin Olivera
}

\section{To cite this version:}

Martin Olivera. Introduction aux formes et raisons de la diversité rom roumaine. Etudes Tsiganes, 2010, 38, pp.26. halshs-00586554

\section{HAL Id: halshs-00586554 https://shs.hal.science/halshs-00586554}

Submitted on 17 Apr 2011

HAL is a multi-disciplinary open access archive for the deposit and dissemination of scientific research documents, whether they are published or not. The documents may come from teaching and research institutions in France or abroad, or from public or private research centers.
L'archive ouverte pluridisciplinaire HAL, est destinée au dépôt et à la diffusion de documents scientifiques de niveau recherche, publiés ou non, émanant des établissements d'enseignement et de recherche français ou étrangers, des laboratoires publics ou privés. 


\title{
Introduction aux formes et raisons de la diversité rom roumaine
}

\author{
Martin Olivera* \\ Article publié dans Roms de Roumanie, la diversité méconnue, Etudes tsiganes, n³8, Paris, \\ 2ème trimestre 2009
}

Entre 535000 et 2,5 millions, le nombre de Romi (selon la nouvelle terminologie officielle) en Roumanie varie considérablement. Le premier chiffre correspond aux résultats du recensement national de 2002, le second aux évaluations hautes de certaines ONG. Quelle que soit l'estimation retenue, leur présence apparaît toutefois non négligeable : sur 22 millions d'habitants, ils représenteraient entre $2,7 \%$ et plus de $10 \%$ de la population roumaine devenant alors la première «minorité nationale », devant les Hongrois ${ }^{1}$.

Les Roms ne sont ainsi jamais invisibles. Dans la rue comme dans les médias, leur existence publique est un fait manifeste du paysage roumain. Omniprésence ne rime cependant pas avec homogénéité, d'où la difficulté à les recenser précisément. Il est même délicat, pour ne pas dire totalement inapproprié, de parler de la «communauté rom de Roumanie » au singulier : le pluriel s'impose à quiconque se fait un temps soit peu attentif aux réalités locales.

Cette diversité apparaît tout autant sociale que culturelle et historique. La Roumanie est un pays récent dans sa forme actuelle (moins d'un siècle), les disparités régionales demeurent aujourd'hui encore prononcées. Entre les grandes villes et les provinces essentiellement rurales le contraste apparaît souvent bien marqué. Mais de non moins importantes différences existent entre les régions provinciales elles-mêmes, témoignages d'une histoire moderne et contemporaine diversifiée.

Or, de ce point de vue, les communautés roms ne constituent pas une population à part, se tenant ou tenue à l'écart des évolutions historiques nationales et régionales. Plus encore, on pourra constater que s'attacher à saisir la diversité rom en Roumanie revient à mieux comprendre l'histoire de ce pays et de ses habitants, n'en déplaise aux nationalistes de tous bords. Mais avant cela, faisons-nous définitivement à l'idée qu'un regard fin et différencié s'impose, par l'analyse de quelques données quantitatives. Ces dernières n'arrivent en effet pas à masquer, lorsqu'on entre dans le détail, l'hétérogénéité du réel.

\section{Les mesures indirectes de la diversité}

Les indicateurs socio-économiques sont bien souvent la meilleure manière d'aborder les Roms comme une population globalement marginale et mal insérée dans la société roumaine. De ce point de vue, ils paraissent former une catégorie homogène, au moins par défaut $^{2}$. L'analyse plus détaillée des résultats des grandes enquêtes quantitatives permet néanmoins de mettre à jour la nécessité de prendre en compte la diversité. Je me baserai ici sur deux sources de matériaux : les résultats du recensement national de 2002 d'une part, et

\footnotetext{
*Ethnologue, Docteur de l'Université Paris X, membre de l'Observatoire européen Urba-rom.

${ }^{1}$ On rappellera à titre de comparaison que les Tsiganes seraient en France autours de 400 000, soit 0,80\% de la population.

2 Cf. par exemple Zamfir, (1993), mais également les divers rapports de la Banque Mondiale et autres organismes internationaux, privés ou publics : Conseil de l'Europe, PNUD, Open Society Institute, OSCE, etc.
} 
l'enquête Pro-Romi sur la «pauvreté des communauté roms de Roumanie » menée en 2005 par l'Agence Nationale pour les Roms ${ }^{3}$ d'autre part.

Le recensement de 2002 est le dernier en date. Conformément à la constitution roumaine de 1991, les citoyens peuvent se déclarer membres de l'une des vingt «minorités nationales $\gg{ }^{4}$.

Sur une population totale de 21680974 habitants, 535140 personnes se sont affirmées Romi. Ce chiffre est certainement inférieur à la réalité : un bon nombre de Roms se déclarent plus volontiers Hongrois ou Roumains, selon les régions. L'explication usuelle à cet écart entre la réalité et l'affirmation publique de l'ethnicité consiste à évoquer une tenace «honte de la tsiganitude »: «Pour de multiples raisons, beaucoup de Rroms évitent de se déclarer comme tels. [...] Beaucoup rechignent à voir souligner une appartenance que par ailleurs ils cherchent à estomper. $»^{5}$

Avoir remplacé ţigan (terme généralement péjoratif en roumain) par le politiquement correct Rom $^{6}$ ne semble toutefois pas avoir eu d'effet radical sur le taux d'intéressés revendiquant leur appartenance «ethnique ». En 1992, les ţigani déclarés étaient certes moins nombreux (401 087) mais, en se basant sur une estimation moyenne d'un million de Roms dans le pays, constatons que le changement de terminologie et, officiellement, d'attitude des institutions à leurs égards, n'apparaît pas décisif pour la grande majorité de ceux ne s'étant déjà pas déclaré ţigani dix ans auparavant ${ }^{7}$.

On rencontre par ailleurs nombre de Roms jugés traditionnels, et portant ostensiblement leur «tsiganitude » (notamment par leurs vêtements), qui avouent s'être déclarés Roumains ou Hongrois. Il n'y a pas là une honte de l'appartenance communautaire, quotidiennement et publiquement affirmée, mais plutôt l'expression d'une distinction essentielle entre le Nous de la communauté locale et les «autres ţigani»: ceux, fantasmés, des médias, de la capitale, d'une autre région, etc. Peu importe alors le terme, Romi et ţigani renvoient à leurs yeux à une seule et même altérité, abstraite et négative, dont ils n'estiment aucunement faire partie.

Inversement, des individus que rien ne distingue de leurs voisins non-Roms, parfaitement insérés dans leur environnement, diplômés de la faculté et non locuteurs du romanès, s'affirment résolument Romi et sont actifs dans des mouvements associatifs et militants sur la question.

Selon le contexte local, les intéressés jugent ainsi opportun de se dirent ou non Romi, pour des raisons diverses et, parfois, contradictoires. Il est en ce sens difficile d'élaborer un «type » de Roms se déclarant plus volontiers Romi devant un formulaire : la vague distinction entre « traditionnels » et « acculturés » (ou « roumanisés »), fréquemment mobilisée en Roumanie, ne paraît elle-même pas pertinente. Les limites de ce recensement sont dès lors une expression de la diversité des situations locales.

Quoiqu'il en soit, l'analyse des résultats n'en demeure pas moins instructive, tant que l'on ne perd pas de vue le caractère tronqué de l'image observée.

\footnotetext{
${ }^{3}$ Résultats du recensement disponibles sur le site de l'INSSE : http://www.insse.ro. L'étude sur la pauvreté des communautés roms de Roumanie (Comunitatile de Romi din România - O hartă a saraciei comunitare prin sondajul PROROMI) est publiée sur le site de l'ANR, http://www.anr.gov.

${ }^{4}$ Hongrois, Roms, Albanais, Arméniens, Bulgares, Tchèques, Croates, Grecs, Juifs, Allemands, Italiens, Macédoniens, Polonais, Russes-Lipovènes, Ruthènes, Serbes, Slovaques, Tatars, Turcs et Ukrainiens.

${ }^{5}$ B. Houliat (1999), p. 20.

${ }^{6}$ Entre Romi et Rromi l'orthographe du mot varie depuis une quinzaine d'années, dans les documents officiels eux-mêmes : actuellement, le simple « $\mathrm{r} »$ semble l'avoir emporté auprès des institutions.

${ }^{7}$ La hausse entre 1992 et 2002 est avant tout à mettre au compte des évolutions sociopolitiques du pays, le climat national étant plus serein une quinzaine d'années après la Révolution qu'en 1992.
} 
Le premier enseignement est une répartition non homogène des Roms sur l'ensemble du territoire national. Les résultats de 2002 sont à cet égard très comparables à ceux de $1930^{8}$. Les départements moldaves, la Dobrogea (Constanţa/CT et Tulcea/TL) mais également le nord valaque (Gorj/GJ, Vâlcea/VL, Arges/AG) semblent moins concernés par la présence rom, tandis que la Transylvanie et le sud valaque (Munténie) affichent des proportions souvent bien supérieures à la moyenne nationale de 2,64\%. Ces taux variables reflètent l'histoire diversifiée des régions qui composent la Roumanie actuelle, on y reviendra.

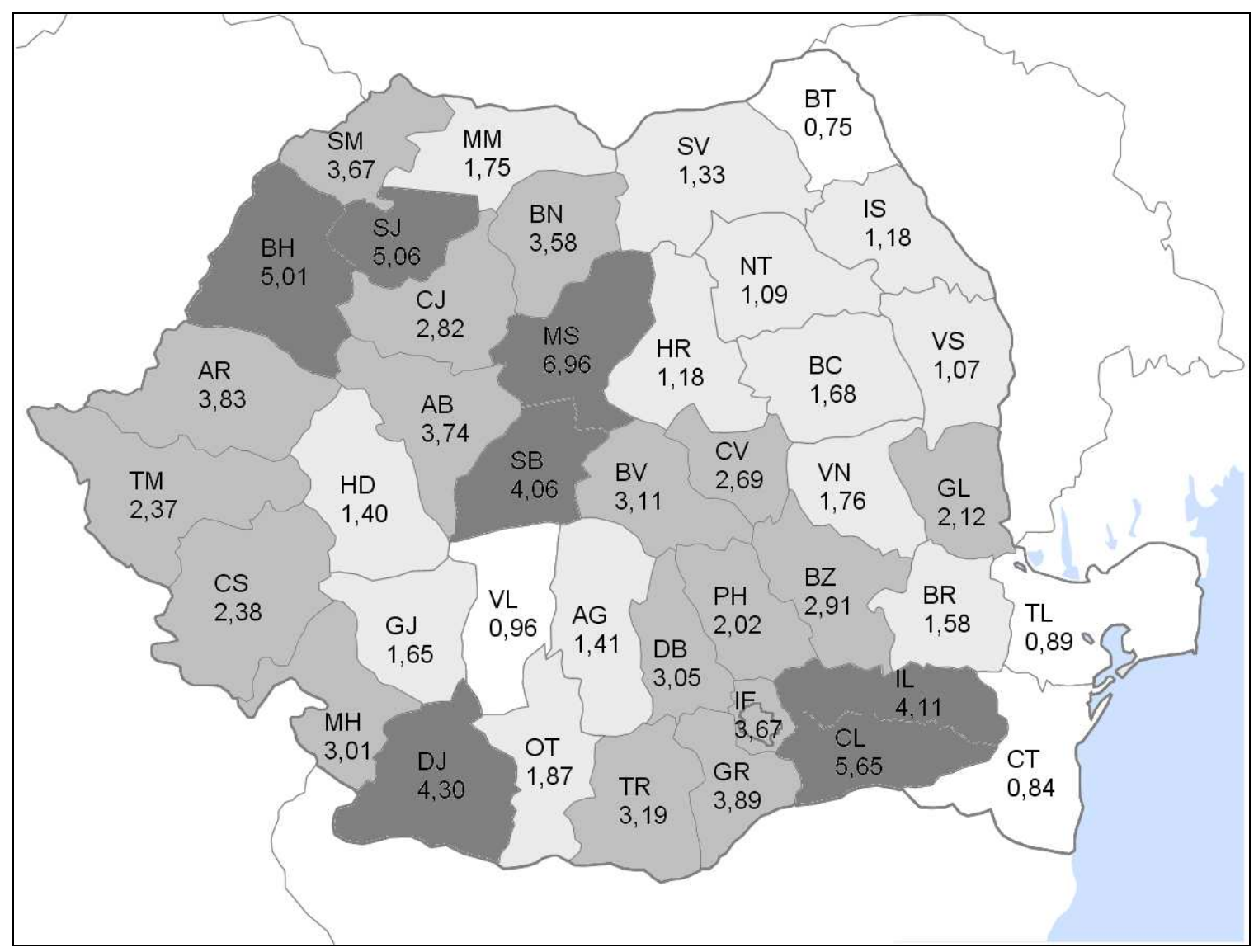

Figure 1. Pourcentage de Romi par département (judeţ)

Sur 535140 personnes à s'être identifiées comme Romi, 237300 (43\%) ont déclaré avoir le romanès pour langue maternelle. Selon les départements, les situations sont très différenciées : du judeţ de Ialomiţa (IL) où plus de $79 \%$ des Romi déclarés possèdent le romanès comme langue maternelle, au judeţ de Covasna $(\mathrm{CV})$ où ils ne sont que 2,63\%, les résultats se répartissent de manière homogène sur toute l'échelle. Et ça n'est pas nécessairement là où les Romi apparaissent les plus nombreux que les locuteurs du romanès le sont également :

- Caraş-Severin (CS) ne se situe qu'en $30^{\text {ème }}$ position pour le nombre de Romi (7914) mais en $3^{\text {ème }}$ pour la proportion de locuteurs du romanès parmi ceux-ci $(66,20 \%)$;

- Bucarest se trouve en $4^{\mathrm{e} e \mathrm{e}}$ position pour le nombre de Romi (27 322), mais seulement $19,58 \%$ de ceux-ci déclarent le romanès comme langue maternelle ( $38^{\text {ème }}$ rang);

\footnotetext{
${ }^{8}$ Cf. « Populatia după etnie la recensamentile din perioadă 1930-2002 dupa judeţe », in Recensamantul populatiei şi locuintelor 2002.
} 
Ailleurs, d'autres situations posent d'autres questions. Les départements de Braşov (BV) et Arad (AR) semblent a priori très semblables: de tailles comparables, ils comptent respectivement $18313(3,11 \%)$ et 17664 (3,83\%) Romi. Les proportions de locuteurs du romanès apparaissent toutefois bien différentes puisqu'ils sont $16,56 \%$ dans le premier, contre $50,96 \%$ dans le judeţ d'Arad. Transparaît ainsi une variété de réalités locales qu'un regard distant ne permet pas de saisir réellement, encore moins d'analyser.

Les chiffres montrent que les Romi déclarés sont davantage ruraux que le reste de la population: ils ne sont que 39\% d'urbains contre une moyenne nationale de 53\%. Aujourd'hui encore, une bonne partie des villes provinciales de Roumanie ayant accédé au statut administratif de «ville» (oraş) sous le communisme demeurent néanmoins marquées par la vie campagnarde ${ }^{9}$. En ce sens, la répartition des Roms ne fait que souligner un trait national : le pays est empreint de ruralité. «Avec plus de 10 millions de ruraux et plus de 3,3 millions d'actifs agricoles, la Roumanie est rurale, paysanne et villageoise, malgré le violent épisode socialiste qui a voulu la fin des paysans », écrit V. Rey. ${ }^{10}$ Le fossé entre la vie urbaine des grands centres historiques (Bucarest, Iaşi, Cluj, Timişoara) et le reste des provinces demeure certain. Mais, là encore, pas de généralité définitive à tirer à propos des Roms : s'ils sont davantage présents à la campagne, on trouve de très anciennes communautés en milieu urbain (cf. l'exemple de Buzău dans ce numéro).

Concernant le profil religieux des Romi, on ne peut que constater sa fidélité au profil national : $82 \%$ se déclarent orthodoxes (pour $86 \%$ de la population totale), 3,74\% catholiques $(4,7 \%), 3 \%$ réformés $(3,2 \%), 1,12 \%$ Gréco-catholiques $(0,88 \%), 0,75 \%$ Baptistes $(0,98 \%)$ et $0,75 \%$ adventistes du $7^{\text {ème }}$ jour $(0,42 \%)$. Seul écart significatif avec la population globale, la proportion de Pentecôtistes : elle est de 6,34\% chez les Romi contre 1,5\% au niveau national. Mais ils ne constituent qu'un peu plus de 10\% du total des Pentecôtistes (34 449 sur 324 462), difficile donc de parler de religion « ethnique ».

D'un point de vue socioprofessionnel, le contraste avec le reste de la population semble davantage marqué :

- 34,31\% de ceux déclarés Romi n'ont pas terminé de cursus scolaire (c-a-d qu'il n'ont pas passé ou obtenu leur examen de capacitate, à la sortie de la $8^{\text {ème }}$ classe, équivalent du BEPC français), contre 5,57\% au niveau national ;

- $25,6 \%$ de Romi se disent analphabètes (20\% des hommes, $30 \%$ des femmes), contre $2,6 \%$ pour la population totale ;

- $\quad 28 \%$ des Romi actifs sont déclarés chômeurs, contre $11,5 \%$ de moyenne nationale ${ }^{11}$;

Les Romi apparaissent globalement dix fois moins alphabétisés que la population globale, près de six fois moins scolarisés, mais en comparaison le ratio pour les chômeurs n'est plus qu'un peu moins de un pour trois. Il n'y a donc proportionnellement pas de congruité entre le

\footnotetext{
${ }^{9}$ On compte en Roumanie une vingtaine de villes de plus de 100000 habitants, totalisant elles-mêmes 6,6 millions d'habitants, soit $30 \%$ de la population. Selon les chiffres officiels, $47 \%$ de la population vit en milieu rural. Si l'on y ajoute les habitants des petites villes provinciales dont les habitants, en grande partie originaires des campagnes alentours, gardent des liens forts et réguliers avec la vie rurale, la proportion atteint donc les $70 \%$.

${ }^{10}$ Suite du paragraphe : «La modernité, toile de fond des dynamiques sociale et économique, peut-elle se penser dans un tel contexte de ruralité ? Depuis le XIXe siècle cette question est au cœur des interrogations sur le devenir du pays. Deux citations célèbres ont peut-être trop fixé les termes du débat, celle de L. Blaga sur "la capacité du village roumain à boycotter l'histoire" (1936) et celle de C. Noica : "nous ne voulons plus être les éternels villageois de l'histoire" (1943)», in V. Rey (2000), p. 48.

${ }^{11}$ Pour relativiser, voici les chiffres réciproques : $65,69 \%$ d'entre eux ont quitté l'école après avoir terminé un cursus d'au moins 8 années ; 74,4\% savent lire et écrire ; 72\% des actifs exercent une activité professionnelle.
} 
bas niveau d'instruction et l'inactivité - sans compter que sont comptabilisés comme inactifs les travailleurs au noir, nombreux en Roumanie, en particulier dans les milieux populaires et ruraux.

Entre Bucarest (où 12,8\% des Romi recensés sont analphabètes) et le département de Harghita (HR) où ils représentent $42,4 \%$, les résultats par judeţ montre par ailleurs la diversité des situations locales. Une vingtaine de départements (essentiellement en Moldavie et dans le sud valaque) présentent des taux équivalents ou supérieurs à $30 \%$ d'analphabètes parmi les Romi. Logiquement, ce sont dans ces mêmes judeţe que la population globale apparait ellemême moins bien alphabétisée (cf. Giurgiu/GG, 7,7\%; Teleorman/TL, 7,1\%). Mais les « exceptions » ne sont pas rares, dans un sens comme dans l'autre : la proportion moyenne selon laquelle les Romi sont dix fois moins alphabétisés que la moyenne nationale n'est pas vérifiée dans la moitié des cas, et les écarts sont parfois importants ${ }^{12}$.

Enfin, concernant l'activité professionnelle on rencontre des écarts significatifs avec le profil national : seulement 33\% des Romi actifs recensés sont salariés (contre $70 \%$ des actifs au niveau national) et plus de 53\% se déclarent «patrons, entrepreneurs privés, artisans ou travailleurs agricoles à leur propre compte » (28\% pour la population active globale). Cette différence peut en partie être expliquée par les difficultés d'accès à l'emploi salarié pour certains Roms depuis la fin du communisme (notamment en milieu rural). Mais on peut aussi y voir pour beaucoup d'autres l'expression d'une éthique économique particulière, relevée à maintes reprises par divers auteurs : les activités recherchées et valorisées par bien des Roms sont basées sur l'indépendance et la maîtrise du temps de travail (commerce, artisanat, activité indépendante etc. $)^{13}$.

\footnotetext{
${ }^{12}$ Damboviţa (DB) : 19,7\% de Romi analphabètes contre 3,4\% de moyenne départementale ; Bistriţa-Năsăud (BN) : 15,8\% de Romi analphabètes contre 2,5\% de moyenne départementale.

${ }^{13}$ Cf. Notamment Reyniers (1998), Formoso (1986), Piasere (1985), Stewart (1994), Olivera (2007).
} 


\begin{tabular}{|c|c|c|c|c|c|}
\hline Judet & Population totale & Romi & $\begin{array}{l}\text { \% de la pop. } \\
\text { locale }\end{array}$ & $\begin{array}{l}\text { Romanès langue } \\
\text { maternelle }\end{array}$ & $\begin{array}{l}\text { \% locuteurs } \\
\text { romanès }\end{array}$ \\
\hline Mureş MS & 580851 & 40425 & 6,96 & 22709 & 56,18 \\
\hline Dolj DJ & 734231 & 31544 & 4,30 & 21254 & 67,38 \\
\hline Bihor BH & 600246 & 30089 & 5,01 & 18177 & 60,41 \\
\hline Bucarest B & 1926334 & 27322 & 1,42 & 5349 & 19,58 \\
\hline Cluj CJ & 702755 & 19834 & 2,82 & 9370 & 47,24 \\
\hline Călăraşi CL & 324617 & 18343 & 5,65 & 4791 & 26,12 \\
\hline Braşov BV & 589028 & 18313 & 3,11 & 3033 & 16,56 \\
\hline Arad AR & 461791 & 17664 & 3,83 & 9001 & 50,96 \\
\hline Sibiu SB & 421724 & 17125 & 4,06 & 3782 & 22,08 \\
\hline Prahova PH & 829945 & 16781 & 2,02 & 5899 & 35,15 \\
\hline Damboviţa DB & 541763 & 16534 & 3,05 & 6814 & 41,21 \\
\hline Timiş TM & 677926 & 16084 & 2,37 & 10433 & 64,87 \\
\hline Buzau BZ & 496214 & 14446 & 2,91 & 6645 & 46,00 \\
\hline Alba AB & 382747 & 14306 & 3,74 & 3974 & 27,78 \\
\hline Teleorman TR & 436025 & 13898 & 3,19 & 7818 & 56,25 \\
\hline Satu Mare SM & 367281 & 13478 & 3,67 & 2520 & 18,70 \\
\hline Galaţi GL & 619556 & 13151 & 2,12 & 5805 & 44,14 \\
\hline Sălaj SJ & 248015 & 12544 & 5,06 & 6726 & 53,62 \\
\hline Ialomiţa IL & 296572 & 12182 & 4,11 & 9661 & 79,31 \\
\hline Bacau BC & 706623 & 11839 & 1,68 & 6536 & 55,21 \\
\hline Giurgiu GG & 297859 & 11573 & 3,89 & 3977 & 34,36 \\
\hline Bistrita Năsăud BN & 311657 & 11155 & 3,58 & 3810 & 34,16 \\
\hline Ilfov IF & 300123 & 11002 & 3,67 & 2889 & 26,26 \\
\hline Iaşi IS & 816910 & 9624 & 1,18 & 5836 & 60,64 \\
\hline Mehedinţi MH & 306732 & 9230 & 3,01 & 5791 & 62,74 \\
\hline Argeş AG & 652625 & 9227 & 1,41 & 1175 & 12,73 \\
\hline Suceava SV & 688435 & 9186 & 1,33 & 2581 & 28,10 \\
\hline Olt OT & 489274 & 9137 & 1,87 & 5178 & 56,67 \\
\hline Maramureş MM & 510110 & 8913 & 1,75 & 4381 & 49,15 \\
\hline Caraş-Severin CS & 333219 & 7914 & 2,38 & 5239 & 66,20 \\
\hline Hunedoara HD & 485712 & 6823 & 1,40 & 1392 & 20,40 \\
\hline Vrancea VN & 387632 & 6813 & 1,76 & 4273 & 62,72 \\
\hline Gorj GJ & 387308 & 6399 & 1,65 & 3168 & 49,51 \\
\hline Neamţ NT & 554516 & 6036 & 1,09 & 2436 & 40,36 \\
\hline Constanţa CT & 715151 & 6023 & 0,84 & 3410 & 56,62 \\
\hline Covasna CV & 222449 & 5973 & 2,69 & 157 & 2,63 \\
\hline Braila BR & 373174 & 5885 & 1,58 & 2473 & 42,02 \\
\hline Vaslui VS & 455049 & 4873 & 1,07 & 2878 & 59,06 \\
\hline Vâlcea VL & 413247 & 3955 & 0,96 & 2212 & 55,93 \\
\hline Harghita HR & 326222 & 3855 & 1,18 & 1238 & 32,11 \\
\hline Botoşani BT & 452834 & 3390 & 0,75 & 1754 & 51,74 \\
\hline Tulcea TL & 256492 & 2272 & 0,89 & 755 & 33,23 \\
\hline $\begin{array}{l}\text { TOTAL ou } \\
\text { moyenne }\end{array}$ & 21680974 & 535140 & 2,64 & 237300 & 43,48 \\
\hline
\end{tabular}

Figure 2. Classement du nombre de Romi par județ et locuteurs du romanès Recensement de 2002 
Les résultats du recensement de 2002 ne permettent pas d'entrer dans les détails audelà du niveau départemental. Directement ou en creux, ces chiffres révèlent toutefois la nécessité de prendre en compte une grande variété de situations régionales et locales. Probablement confrontée au même constat, l'Agence Nationale pour les Roms de Roumanie $(A N R)^{14}$ mena en 2005 une étude plus détaillée afin de mesurer au mieux la «pauvreté des communautés roms » du pays. Au-delà des résultats directs de cette vaste enquête, l'examen du protocole mis en œuvre est, en lui-même, fort instructif.

Dans son propos introductif, l'étude détaille ses trois objectifs :

- « construire une typologie et le profil des communautés de Roms d'après leur état de pauvreté/aisance ;

- localiser les communautés de Roms d'après leur type d'implantation et par région en fonction de leur degré de pauvreté et de leur mode de vie ;

- hiérarchiser les problèmes sociaux de ces communautés avec l'objectif d'orienter l'intervention sociale et les actions de développement. »

Sont définies comme «communautés » les implantations locales de vingt foyers ou plus. L'étude en a recensé 848 (dans 549 localités), pour lesquelles un questionnaire détaillé fut complété par des acteurs locaux. Ceux-ci devaient idéalement être au nombre de trois : un « représentant de la communauté rom locale », un représentant de la mairie «d'origine rom ou connaissant les problèmes des Roms » et un représentant (coopté par les deux précédents) d'une autre organisation locale (par exemple membre d'une ONG ou un médiateur) ${ }^{15}$.

D'après les résultats, la dimension moyenne d'une «communauté » est de 300 personnes, la dimension médiane de 170 personnes. Plus de 60\% des Roms ainsi recensés vivent dans des communautés locales de plus 500 membres.

$60 \%$ des communautés identifiées se trouvent en milieu rural. Le chiffre atteint 76,2\% si l'on ajoute les petites villes provinciales de moins de 30000 habitants. La répartition par type de localité se fait comme suit :

- commune $^{16}$ pauvre : $10,2 \%$

- commune moyennement développée : $20,2 \%$

- commune développée : $28,9 \%$

- ville de moins de $30000 \mathrm{~h}: 16,2 \%$

- ville entre 30000 et $100000 \mathrm{~h}: 13 \%$

- ville entre 100000 et $200000 \mathrm{~h}: 3,5 \%$

- ville de plus de $200000 \mathrm{~h}: 8 \%$

Trois séries d'indicateurs sont utilisés pour mesurer le degré de pauvreté d'une communauté cf. tableau ci-dessous.

\footnotetext{
14 «L'Agence Nationale pour les Roms est une structure gouvernementale de représentation des Roms au niveau national. L'institution fonctionne comme organe spécifique de l'administration publique centrale [...] L'Agence est dirigée par un président ayant rang de secrétaire d'État, nommé par décision du premier ministre. [...] L'Agence coordonne et évalue les mesures dans les domaines de l'intervention sociale relevant de la stratégie du gouvernement afin d'améliorer la situation des Roms [...]», présentation de l'agence sur le site internet http://www.anr.gov.ro , ma traduction.

${ }^{15}$ Le document spécifie : «Chaque fois que cela est possible, deux de ces trois personnes au moins doivent être d'ethnie rom et reconnaître leur appartenance ethnique. » Au passage, l'étude fournit trois estimations de la population roumaine s'identifiant comme rom : variante basse : 730174 ; variante moyenne : 851048 ; variante haute : 968275 .

${ }^{16}$ En roumain, le terme «commune » (comună) désigne une localité rurale, par opposition à oraş (ville) et mипicipiu (ville de plus de 40000 habitants).
} 


\begin{tabular}{|c|c|c|}
\hline \multirow[t]{3}{*}{$\begin{array}{l}\text { Où vivent-ils ? } \\
\text { Accessibilité }\end{array}$} & $\begin{array}{l}\text { En marge de la localité (1 oui, } 0 \\
\text { non) }\end{array}$ & \multirow{3}{*}{$\begin{array}{l}\text { Une communauté est considérée } \\
\text { comme souffrant de problèmes } \\
\text { d'accès si l'un des trois indicateurs } \\
\text { a une valeur de } 1 .\end{array}$} \\
\hline & $\begin{array}{l}\text { Route d'accès modernisée - pavée } \\
\text { ou asphaltée ( } 1 \text { non, } 0 \text { oui })\end{array}$ & \\
\hline & $\begin{array}{l}\text { Proximité de dépôt d'ordures (1 } \\
\text { oui, } 0 \text { non) }\end{array}$ & \\
\hline \multirow[t]{2}{*}{$\begin{array}{l}\text { Comment vivent-ils ? } \\
\text { Infrastructures }\end{array}$} & $\begin{array}{l}\text { Plus de } 50 \% \text { des foyers n'ont pas } \\
\text { d'accès direct à de l'eau potable ( } 1 \\
\text { oui, } 0 \text { non) }\end{array}$ & \multirow{2}{*}{$\begin{array}{l}\text { Une communauté est considérée } \\
\text { comme souffrant de problèmes } \\
\text { d'accès aux infrastructures } \\
\text { électriques et de distribution d'eau } \\
\text { si au moins l'un des deux } \\
\text { indicateurs a une valeur de } 1 \text {. }\end{array}$} \\
\hline & $\begin{array}{l}\text { Plus de } 50 \% \text { des foyers ne sont pas } \\
\text { connectés au réseau d'électricité ( } 1 \\
\text { oui, } 0 \text { non) }\end{array}$ & \\
\hline $\begin{array}{l}\text { Quelles ressources ont-ils? } \\
\text { Sources de revenus }\end{array}$ & $\begin{array}{l}\text { La principale source de revenu est } \\
\text { le revenu minimum garanti (RMI) } \\
\text { ou des activités occasionnelles }\end{array}$ & $\begin{array}{l}\text { Une communauté est considérée } \\
\text { comme présentant des problèmes } \\
\text { sérieux si plus de } 50 \% \text { des foyers } \\
\text { n'ont pas d'autres sources de } \\
\text { revenus que le revenu minimum } \\
\text { garanti ou des activités } \\
\text { occasionnelles (code valeur } 1 \text { ). }\end{array}$ \\
\hline
\end{tabular}

Figure 3. Indices utilisés pour la construction de la typologie des communautés ${ }^{17}$

Une communauté est considérée comme rencontrant :

- des «problèmes très sévères » (HIGHPROB) si des problèmes sont identifiés dans chacun des trois grandes champs (accessibilité, infrastructures, sources de revenus) : $14 \%$ des communautés recensées relèvent de cette classe ;

- des «problèmes sévères » (MIDPROB) si elle réunit deux indices problématiques : $45 \%$ des communautés recensées ;

- des «problèmes légers » (LOWPROB) si elle ne rencontre des difficultés que dans un seul des trois champs : $31 \%$ des communautés ;

Si tous les indicateurs ont une valeur égale à zéro, la communauté est considérée comme ne rencontrant pas de problème particulier (NONPROB - fără probleme deosebite) : $10 \%$ des communautés recensées sont dans cette situation.

Une communauté est classifiée comme «pauvre » (săracă) si elle entre dans l'une des deux premières catégories («problèmes très sévères » et «problèmes sévères »). Selon cette méthode, près de $60 \%$ des communautés recensées sont « pauvres ». Elles abritent un peu plus de $50 \%$ de la population rom ainsi identifiée et se situent en grande majorité dans des communes rurales ou des villes de moins de 30000 habitants $(74 \%)$.

$61 \%$ des membres des communautés classifiées comme présentant des «problèmes très sévères » possèdent le romanès comme langue principale. Difficile toutefois d'établir une corrélation entre "pauvreté communautaire » et langue maternelle puisque les proportions varient aléatoirement d'une catégorie à l'autre, cf. tableau ci-dessous.

On observe en revanche un lien direct entre les conditions de vie et la proportion d'émigrants (passés ou présents): ce ne sont pas les membres des groupes les plus défavorisés qui émigrent, de même que le fruit de la migration permet souvent d'améliorer les conditions de vie, de retour au pays.

\footnotetext{
${ }^{17}$ D'après « Tablelul 4. Indicatori pentru constructia tipologica », p. 16 de l'étude, ma traduction.
} 


\begin{tabular}{|l|c|c|c|c|c|}
\hline & NONPROB & LOWPROB & MIDPROB & HIGHPROB & Total \\
\hline $\begin{array}{l}\text { \% de personnes ayant } \\
\text { au moins suivi une } \\
\text { scolarité primaire }\end{array}$ & 65,3 & 61,3 & 58,6 & 45,1 & 58,6 \\
\hline$\%$ d'anciens émigrés & 12,4 & 10,4 & 8,9 & 5,1 & 9,4 \\
\hline$\%$ d'émigrés actuels & 6,9 & 6,4 & 4,3 & 2,8 & 5,2 \\
\hline $\begin{array}{l}\% \text { de personnes ayant } \\
\text { le romanès comme } \\
\text { langue principale }\end{array}$ & 48 & 42 & 38 & 61 & 44 \\
\hline $\begin{array}{l}\text { Nombre moyen de } \\
\text { personnes par foyer }\end{array}$ & 4,33 & 4,57 & 4,77 & 4,80 & 4,67 \\
\hline
\end{tabular}

Figure 4. Profil des populations vivant dans les communautés roms ${ }^{18}$

Comme le précise le texte de l'analyse, les indicateurs utilisés ne permettent pas de mesurer la pauvreté au niveau individuel ou familial mais seulement collective. L'étude s'intéressant uniquement aux implantations relativement importantes, il est d'autre part peu surprenant d'y trouver une majorité de groupes spatialement marginalisés et, de ce fait, confrontés à des difficultés d'accès aux infrastructures d'eau et d'électricité.

À noter que plus de 500 questionnaires ont été éliminés de l'analyse car émanant de communautés «non compactes », disséminées parmi les populations avoisinantes (« répandus parmi d'autres groupes ethniques », p. 12), tandis que 253 autres ont été rejetés en raison de la dimension trop faible de la communauté (moins de 20 foyers). Au total, le nombre de cas écartés dépasse celui pris en compte (pour diverses raisons, 985 questionnaires n'ont pas été retenus).

En dépit du filtre spécifique de cette étude, laissant de côté un bon nombre de groupes probablement mieux insérés dans le tissu local, remarquons que les résultats apparaissent déjà nuancés : toutes les communautés ne sont pas confrontées aux mêmes difficultés, ni dans les mêmes proportions.

Tout comme le faisaient apparaître les résultats du recensement de 2002, les situations sont par ailleurs diversifiées selon les territoires. C'est en Munténie que se situent le plus de communautés compactes ainsi recensées $\left(27,1 \%\right.$ du total, sur dix départements $\left.{ }^{19}\right)$, mais elles sont également nombreuses en Crişana (Arad et Bihor, 10,3\%) et en Transylvanie centrale (Cluj et Mureş, 8,8\%). Pour ces deux derniers départements, on constate toutefois un écart avec les chiffres du recensement de 2002, selon lequel y vivent plus de $11 \%$ du total des Romi du pays : on peut en déduire un plus grand éparpillement de ceux-ci dans le tissu local, en communautés de moins de 20 foyers. Inversement, les judeţe moldaves de Suceava, Neamţ, Bacău et Vrancea totalisent plus de $8 \%$ des communautés de l'étude, alors qu'ils ne comptent que pour $6 \%$ du total des Romi. Des raisons historiques régionales permettent d'interpréter cette concentration accrue en communautés importantes ou, inversement, une plus grande dispersion des groupes dans l'environnement, on y reviendra.

Notons enfin que les départements ruraux de Bihor (BH), Salaj (SJ) et Mureş (MS), marqués par une importante présence hongroise (respectivement $26 \%, 23 \%$ et $40 \%$ de la population en 2002), abritent une bonne partie des communautés classifiées comme «HIGHPROB » : les trois judeţe comptent à eux seuls 43 des 118 communautés ainsi identifiées.

Et nous voilà ramenés à la nécessité d'un regard fin et nuancé sur les réalités roms en Roumanie, prenant en compte le contexte socio-historique local et régional. L'approche

\footnotetext{
${ }^{18}$ D'après « Tabelul 10. Un profil social al populatiei locuind în comunitatile de romi sarace », p. 21.

${ }^{19}$ AG, DB, PH, BZ, BR, IL, CL, GG, TR, IL.
} 
globale quantitative met certes en relief un fait incontestable : en proportion, une bonne partie des citoyens roumains peu ou mal insérés dans la vie «moderne » et vivant dans des conditions matérielles difficiles sont roms. Les analphabètes Romi représentent $20 \%$ du total des analphabètes recensés, ils sont 3,4\% des chômeurs et $13 \%$ de ceux n'ayant terminé aucun cursus scolaire. Mais de là à considérer «la minorité rom » comme une entité homogène, globalement «pauvre» et «marginale», il y a un pas considérable que les chiffres euxmêmes n'arrivent pas à justifier.

La situation économique s'est considérablement dégradée pour nombre de Roms au cours des vingt dernières années, mais cela est également vrai pour une bonne partie des Roumains (ou Hongrois, ou autre), particulièrement en milieu rural et dans les petites villes provinciales : fermeture des fermes collectives, des entreprises d'État, détérioration des services publics de santé et d'instruction, etc.

De ce point de vue, la proportion de Roms roumains émigrés en Europe occidentale correspond, selon diverses estimations, à la moyenne nationale de $10 \%$ d'émigrés ${ }^{20}$. Là encore, la situation des communautés roms parait indissociable du contexte socio-économique national et local. Il est, en d'autres termes, impossible d'aborder les Roms de Roumanie comme une «classe sociale» distincte. Mais il est également bien difficile de les regarder comme une « minorité culturelle » homogène.

À cet égard, un dernier élément de l'enquête pro-Romi est instructif. Il concerne ce que les enquêteurs dénomme l' «appartenance culturelle» des communautés roms. Le questionnaire proposait en effet de rattacher la communauté locale à des " groupes culturels » (sans que l'on sache comment ceux-ci ont été arrêtés et définis) ${ }^{21}$. Sur les 848 communautés recensées, notons que plus de 500 entrent dans les catégories «Autres Roms » et «Sans réponse »: en effet, on verra plus loin qu'un bon nombre de Roms ne s'identifient explicitement à aucun ethnonyme particulier, tout en appartenant à des communautés spécifiques. Pour ceux mobilisant un nom de groupe - ou, plus sûrement, affiliés à un «groupe culturel» par ceux ayant complété le questionnaire - les résultats apparaissent différenciés :

- une majorité des communautés de « cărămidari » («briquetiers » - 35 communautés) est pauvre $(80 \%)$;

- une majorité des communautés «rudari» (Tsiganes roumanophones travaillant traditionnellement le bois - 99 communautés) est pauvre (69\%);

- une majorité des communautés « vătraşi » («Tsiganes du foyer », implantés de longue date sur un territoire, 48 communautés) est pauvre $(65 \%)$;

- une majorité des communautés « autres » (262 communautés) est pauvre (57\%);

- une majorité des «n'ayant pas répondu - indéterminé » (242 communautés) est pauvre $(62 \%)$;

- une majorité des communautés « căldărari » (« chaudronniers »- 67 communautés) est non-pauvre (51\%) ;

\footnotetext{
${ }^{20}$ Cf. figure 4 ci-dessus. Par ailleurs, selon diverses sources, les Roms roumains seraient 8000 à 10000 en France (rapports Romeurope), 25000 en Espagne (M. Pajares), 50000 en Italie (Saletti Salza), 10000 en Belgique (I. Hasdeu), soit approximativement 100000 personnes, correspondant à $10 \%$ de la population rom roumaine. Les Roumains émigrés ces quinze dernières années seraient quant à eux près de 800000 en Espagne, plus de 600000 en Italie, plus de 100000 en Allemagne, 50000 en France, 20000 au Royaume-Uni, soit aux alentours de 2 millions d'individus, équivalant, là encore, à $10 \%$ de la population roumaine totale. Cf. «Romanian diaspora» in Wikipédia.org, http://en.wikipedia.org/wiki/Romanian_diaspora. Il ne s'agit bien entendu que de proportions, les statistiques officiels n'existant généralement pas.

${ }^{21}$ Cf. la question 4 du formulaire : «Quel type de Romi (neam) considèrent être les membres de la communauté (ex. caldarari, rudari etc.) ? 》 (Ce fel de romi (neam) se considera a fi membrii comunitatii (de ex. căldărari, rudari etc.) ?), p. 25.
} 
- une majorité des «Roms roumanisés » (34 communautés) est non-pauvre (53\%) ;

- une majorité des « ursari » (littéralement «montreurs d'ours », i.e. descendants de saltimbanques, 61 communautés) est non-pauvre (57\%).

Au-delà des pourcentages eux-mêmes, assez peu exploitables en l'état ${ }^{22}$, il est intéressant de noter l'intérêt porté par les auteurs de l'étude à la différenciation entre "groupes culturels »: elle dénote une diversité qui, à défaut d'être précisément mesurable dans de telles enquêtes, s'impose aux analystes, bon gré, mal gré. Tandis que l'étude tente d'objectiver au mieux la pauvreté des communautés en entrant dans les détails du local, elle ne peut plus faire l'économie d'une mention des différences socioculturelles entre groupes. Ces dernières n'apparaissent pas anecdotiques et renvoient à des situations hétéroclites.

D'où provient cette diversité socioculturelle ? Pourquoi n'y a t il pas une communauté rom mais des groupes variés, certains se ressemblant et entretenant des relations plus ou moins étroites, d'autres bien différents et s'évitant soigneusement? Comment expliquer la fréquente absence de sentiment d'appartenance «ethnique » et, dans le même temps, l'importance de l'attachement communautaire local ? Enfin, d'où provient cet écart entre des représentations nationales univoques («les Tsiganes, population marginale problématique ») et les réalités locales où le sort des uns (Roms) et des autres (non-Roms) apparaît intimement lié, plus encore en milieu rural ? L'histoire des régions composant la Roumanie d'aujourd'hui permet de répondre à ces questions et d'en éclairer les paradoxes.

\section{La diversité socioculturelle rom : une histoire nationale}

1919, traité de Saint-Germain, naissance de la «Grande Roumanie » (România Mare) telle que nous la connaissons aujourd'hui, par l'unification des anciennes principautés moldovalaques avec la Transylvanie.

Jusqu'alors, les provinces danubiennes de Moldavie et de Valachie (elle-même formée de l'Olténie et de la Munténie) d'une part, à l'est et au sud de l'arc carpatique, et les provinces occidentales (Transylvanie mais également Banat, Crişana, Maramureş et Bucovine) d'autre part, constituaient deux ensembles voisins mais néanmoins distincts, politiquement, économiquement et culturellement (cf. figure 5). Les principautés de Moldavie et Valachie furent longtemps sous influence grecque, ottomane et slave (elles ne formèrent un premier état roumain indépendant qu'en 1859), tandis que la Transylvanie et ses régions voisines étaient davantage tournées vers l'ouest (royaume de Hongrie puis empire habsbourgeois) ${ }^{23}$.

En 1919, les roumanophones sont très largement majoritaires dans les provinces danubiennes (près de $90 \%$ de la population) mais le sont également en Transylvanie, malgré une importante minorité de langue hongroise (localement majoritaire, en particulier dans le pays sicule - départements actuels de Harghita, Covasna et, en partie, Mureş) et la présence d'autres populations : Saxons de langue allemande entre Braşov, Sibiu et Alba-Iulia, Serbes et Croates dans le Banat, Ukrainiens et Ruthènes dans la région de Satu-Mare et le Maramureş,

\footnotetext{
${ }^{22}$ Il apparaît par exemple que « $80 \%$ des communautés de cărămidari sont pauvres », mais ce sont bien souvent des communautés pauvres qui, à défaut d'autre activité possible, fabriquent occasionnellement des briques activité pour laquelle sont seulement nécessaires de la terre et de l'eau. L'ergonyme cărămidari est ainsi rarement un endonyme mais plutôt un exonyme utilisé, précisément, à l'attention de groupes roms défavorisés... Le terme căldărari est quant à lui usité pour désigner diverses communautés perçues comme «traditionnelles » et travaillant le métal, sans qu'il y ait nécessairement de rapports entre les unes et les autres.

${ }^{23} \mathrm{Cf}$. chronologie d'histoire roumaine dans ce même numéro.
} 
etc. De la même manière, les provinces moldo-valaques sont homogènes du point de vue confessionnel, avec une très large majorité de chrétiens orthodoxes, tandis que les confessions se font bien plus variées en Transylvanie: catholiques, réformés, orthodoxes et grécocatholiques (uniates) possèdent souvent leurs lieux de culte les uns à côtés des autres dans un même village.

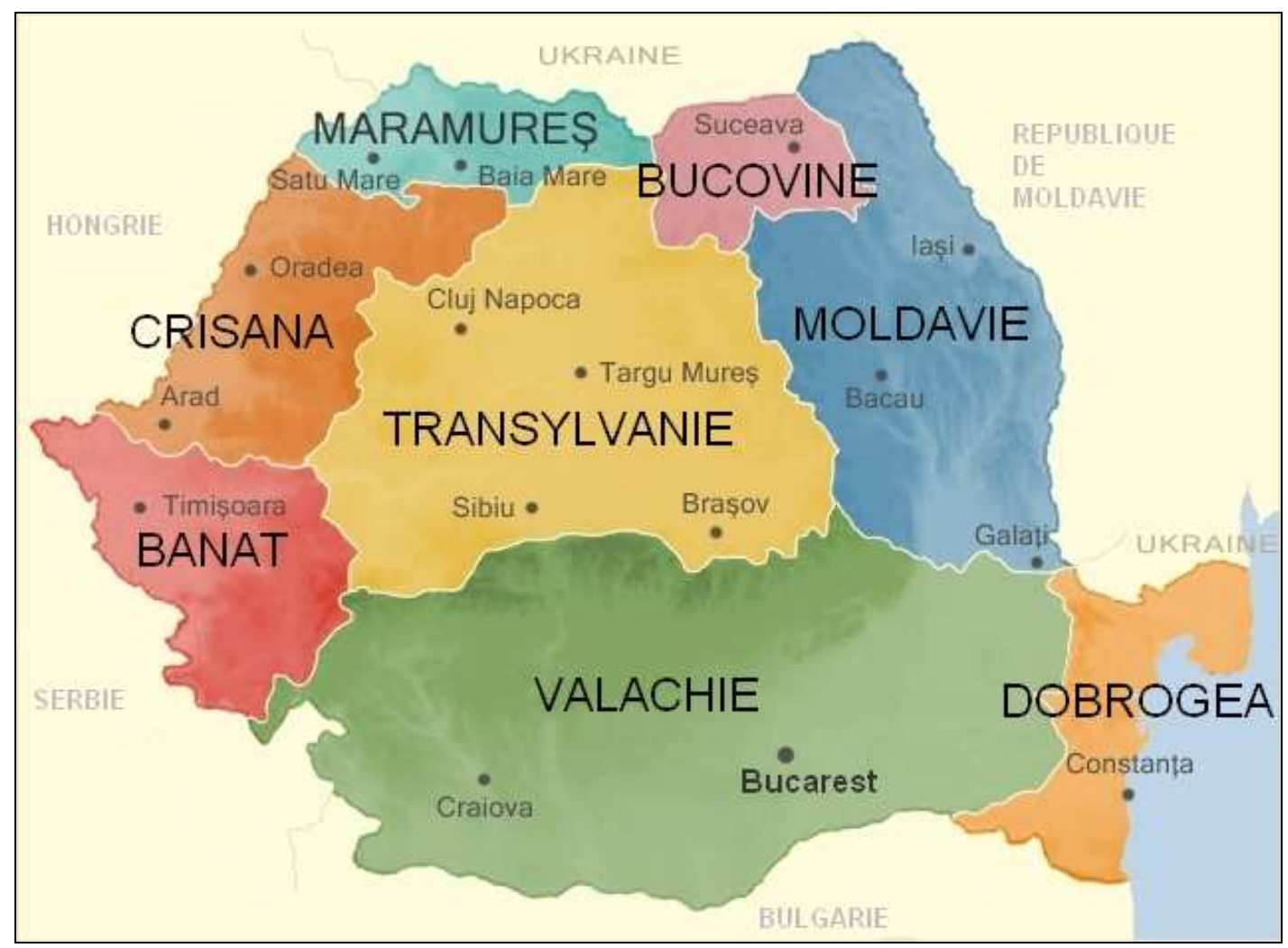

Figure 5. Grandes régions historiques de Roumanie

C'est en Moldavie-Valachie qu'apparaissent ceux que les archives désignent comme atsigani, venus des Balkans dans le courant du XIVe siècle ${ }^{24}$. On assiste alors a de nombreux mouvements de populations dans toute l'Europe balkanique et en Anatolie, liés notamment à la conquête et à l'implantation ottomane (Turcs, Tatares, Arméniens, etc.) ${ }^{25}$. Dans les principautés moldo-valaques, le terme grec atsigan, puis ţigan, servira à désigner une partie de ces populations venues d'ailleurs et réduites sur place en «captivité » (robie) ou esclavage. À leur arrivée, les ţigani sont en effet fixés dans ces territoires qui, pour diverses raisons, semblent manquer de main d'œuvre. Ils sont attribués aux seigneurs locaux (boyards), aux monastères et, pour certains groupes, directement rattachés à la couronne princière. Ces derniers préservent une relative liberté de mouvement, au moins locale, afin, notamment, de pratiquer un artisanat recherché : le travail du métal ${ }^{26}$.

Durant toute l'époque moderne, ţigan correspond donc en ces terres à une catégorie juridique particulière : contrairement aux serfs paysans valaques, appelés vecini en Moldavie et rumâni

\footnotetext{
${ }^{24}$ Le terme tigan est attesté dans les archives valaques pour la première fois en 1385 . Provenant du grec atsiganoi et/ou du turc cingene, il donnera cigàny en hongrois, zigeuner en allemand et, au début du XIXe siècle, Tsigane en français (1826). Sur l'arrivée des ţigani en pays roumains, cf. Achim (2004), pp. 13-20.

${ }^{25}$ Pour un panorama général, cf. Castellan (1991), pp. 29-84.

${ }^{26}$ Sur l'institution de l'esclavage des Tsiganes dans les principautés moldo-valaques, cf. Potra (1939), Painatescu (1941), Vaux de Foletier (1970), Djuvara (1989), Achim (2004) et Piasere (2004).
} 
en Munténie ${ }^{27}$, les ţigani étaient propriété directe de leur maîtres. Ils pouvaient avoir une valeur d'échange et être cédés (en cas de dettes notamment - cf. l'article de P. Petcut dans ce numéro), tandis que les serfs, quoiqu'également attachés à un domaine et non libres de leurs mouvements et activités (corvées etc.), étaient liés à la terre qui est, elle, propriété du seigneur.

Voilà pourquoi l'on parle d'esclavage des Tsiganes dans les principautés moldo-valaques. Il ne s'agit pas d'une traite de type commercial telle que la traite négrière ou celle pratiquée dans le monde arabo-musulman (marchands d'esclaves s'approvisionnant continuellement et vendant leur marchandise à un grand nombre de particuliers) mais d'une fixation rapide et contrainte de groupes familiaux migrants dans des territoires féodaux ${ }^{28}$. Tout en créant une distinction fondamentale, juridique et symbolique, entre tigani et serfs valaques, leur asservissement rapide amènera les dits ţigani à vivre auprès de et avec ces rûmani, dès la fin du XIVe siècle.

En Transylvanie, ceux que l'on appelle cigàny semblent de même rapidement asservis et fixés dans le paysage. Leur condition juridique y est toutefois différente qu'en Valachie et Moldavie puisqu'ils deviennent juridiquement des serfs (jobbàgy) comme les autres. D'autre part, contrairement aux principautés moldo-valaques, la Transylvanie et ses régions voisines (Banat, Crişana, Maramureş) abritent une grande diversité linguistique et culturelle, ce depuis le Haut Moyen-Âge.

La principauté transylvaine fut longtemps dominée par le «règne des trois nations»: hongroise, saxonne et sicule. Les premiers administraient les comitats de l'ouest et du centre de la principauté, les seconds la région entre Braşov et Alba-Iulia, les derniers un territoire accolé aux Carpates correspondant actuellement aux départements de Harghita, Covasna et, pour partie, Mureş. Jusqu'au XIXe siècle, les Valaques, bien que globalement majoritaires dans la principauté, ne formèrent pas une «nation » (ils ne possédaient pas de noblesse) mais la masse du petit peuple (cf. chronologie dans ce numéro).

Selon leur lieu d'implantation, les communautés roms transylvaines furent donc en contact avec diverses populations, dès l'origine. Des échanges de toutes sortes ce sont bien entendu opérés entre les uns et les autres. C'est ainsi qu'on rencontre des Roms saxons dans la région de Sibiu, des Roms hongrois à Cluj, des Gabori autours de Târgu-Mureş etc. ${ }^{29}$ En sus des disparités socio-économiques locales, s'ajoute donc une diversité culturelle et religieuse inconnue en Moldavie et Valachie.

Concernant ces dernières, l'Ancien Régime perdurera jusqu'aux années 1840-1860, selon les régions, époque à laquelle les autorités veulent en finir avec la féodalité : il s'agit en effet d'établir un état national «moderne » sur le modèle occidental, contre les privilèges seigneuriaux et ecclésiastiques. Sont adoptées des réformes agraires qui abolissent définitivement le servage des paysans mais aussi la robie des ţigani ${ }^{30}$.

\footnotetext{
${ }^{27}$ Vecini signifie aujourd'hui «voisin », rumâni a donné... Roumain.

${ }^{28}$ Parmi ces groupes migrants, n'y avait-il que des descendants de populations parties du sous-continent indien quelques siècles auparavant? Difficile, voire impossible, de l'établir. L'origine d'une langue n'est pas mécaniquement celle de ses locuteurs, contrairement à ce que nous enseigne l'idéologie nationale moderne. Notons par ailleurs que la célèbre affiche montrant des «esclaves tsiganes à vendre » (A vinde sclavi ţiganeşti) est à n'en pas douter un faux récemment édité : ni la graphie, ni la langue utilisées ne correspondent à l'époque (1852).

${ }^{29}$ Pour un exemple détaillé d'une telle intégration locale, nourrissant la spécificité d'une communauté rom, cf. Olivera (2009).

${ }^{30}$ Certains groupes familiaux roms partent dès cet époque tenter leur chance ailleurs, en Europe occidentale ou aux Etats-Unis. Ainsi des Roms dits Kalderash de Montreuil qui, après un séjour en Russie à la fin du XIX ${ }^{\text {ème }}$, s'installent en banlieue parisienne dès l'entre-deux-guerres, cf. Williams (1984). Ces Roms sont loin d'être les seuls à participer à ces grandes migrations de 1850-1930 : de nombreuses populations rurales d'Europe centrale et orientale (Pologne, Tchéquie, Hongrie...) émigrent alors vers ce qu'ils espèrent être de meilleurs cieux. Là encore, l'histoire des uns et celle des autres apparaissent indissociables.
} 
Il est d'usage d'appréhender l'asservissement des Roms de Roumanie comme la cause première de leur marginalisation vis-à-vis de la société majoritaire. Marcel Courthiade estime ainsi qu' «[...] à l'issue de cinq siècles d'un pareil traitement, les séquelles marquent profondément les relations Roms/non-Roms dans les terres roumaines [et qu'il] est important de considérer les comportements des groupes sociaux de Roumanie à travers le prisme de cette perception post-esclavagiste du monde. ${ }^{31}$

On peut néanmoins proposer une perspective plus nuancée.

La servitude des ţigani en pays roumains et transylvains aura en effet eu diverses conséquences, pouvant paraître paradoxales :

- D'abord, sur le plan symbolique : lorsque la Roumanie sortira de l'âge féodal au cours du XIX ${ }^{\text {ème }}$ siècle pour s'ériger en nation «moderne », la figure du ţigan (très présente dans la littérature de cette époque, cf. par exemple, dans deux registres différents, I. Budai-Deleanu et $M$. Kogalniceanu) jouera le rôle de vestige honnis de l'ère obscurantiste des boyards. Les ţigani deviennent alors dans les représentations populaires, et demeurent aujourd'hui encore, la part «primitive » et non-civilisée du pays, objet de mépris, de dégoût et, occasionnellement, de pitié voire de fascination. Étant eux-mêmes culturellement nationaux, et ce depuis plusieurs siècles, les Roms partagent cette représentation, non pour eux-mêmes et leur propre communauté, mais envers les autres ţigani. Car il y a toujours d'autres ţigani qui sont, pour le locuteur, très différents de lui-même ;

- En effet, autre conséquence sur le plan social de l'implantation locale des ţigani: on assiste à l'émergence rapide de communautés distinctes les unes des autres, selon leur lieu de vie. Les conditions d'existence étaient très variables d'un domaine à l'autre, suivant la richesse du seigneur ou du monastère, les règles appliquées, les activités professionnelles pratiquées, les aléas locaux, historiques ou naturels, etc. D'autre part, leur nombre important et la diversité des itinéraires familiaux ou personnels a entraîné depuis fort longtemps la mobilité sociale d'une partie des familles et individus ${ }^{32}$;

- Enfin, sur le plan culturel, l'enracinement rapide, contraint ou libre, des ţigani dans des terroirs généralement ruraux aura entraîné des échanges soutenus, volontaires ou non, entre paysans locaux et Roms - ceci étant valable pour les ţigani attachés à un domaine comme pour ceux (en particulier artisans du métal ou chercheurs d'or) qui jouissaient d'une certaine mobilité, au moins saisonnière, dans un territoire donné.

C'est ainsi que nombre de traditions jugées aujourd'hui «authentiquement tsiganes » sont en réalité d'anciennes coutumes locales, autrefois communes aux Tsiganes, Valaques et/ou Hongrois, tombées en désuétude chez les paysans mais toujours pratiquées par les Roms. Les jupes plissées et très colorées des Romnia sont par exemple directement issues des traditions vestimentaires paysannes de la région (idem pour le fichu et les parures de pièces d'or du sud valaque), tout comme l'organisation de la parenté en «lignages » (les viţi, avec terminaison en -eşti), telle qu'on la retrouve dans certains groupes roms, était coutumière dans les communautés paysannes roumaines.

La multitude d'ethnonymes et ergonymes utilisés dans les régions de Roumanie afin de mettre de l'ordre parmi les Tsiganes, reflète en partie leur diversité : căldărari, zlătari, aurari, cărămidari, lăutari, ursari, argintari, cortorari, cuţitari, fierari, cazanji, covaci, lingurari, potcovari, rudari, salahori, spoitori, gabori, badogosi, laieşi, pletoşi, romungre,

\footnotetext{
${ }^{31}$ M. Courthiade (1993), p. 17.

${ }^{32}$ Cf. le cas de Stefan Răzvan, d'origine tsigane, qui fut homme de confiance de Mihai Viteazul et Sigismond Bathory. Il monta même sur le trône de Moldavie durant quelques mois de l'année 1595 - Courthiade (2007).
} 
vatraşi,... autant de termes censés correspondre à des groupes tsiganes différents ${ }^{33}$. La plupart renvoient, directement ou non, à une activité professionnelle: les căldărari font des chaudrons (căldări), les cărămidieri des briques (cărămizi), les lingurări des cuillères en bois (lingură), les covaci sont forgerons (kovàcs en hongrois), les ursari étaient autrefois saltimbanques et dresseurs d'ours, les potcovari maréchaux-ferrants, les cortorari vivent sous la tente (cort) et sont d'ailleurs souvent des căldărari ou des spoitori (rétameurs), les lăutari sont musiciens, etc.

Si tous ces termes ne coïncident pas toujours, loin de là, avec des communautés réelles ou, plus précisément, si plusieurs communautés (n'entretenant aucun rapport les unes avec les autres) sont souvent désignées par un même terme, cette manière d'appréhender les Tsiganes dans les terres roumaines apparaît directement liée à l'histoire de ces contrées. En particulier à l'asservissement des ţigani et à la forme particulière qu'a pu prendre cette servitude, en insistant localement sur les spécialisations artisanales.

Mais deux Roms perçus comme căldărari (car travaillant le métal ou portant des vêtements jugés «traditionnels »), issus de deux régions ou de deux localités éloignées et n'ayant pas de rapport de parenté ne se reconnaîtront sans doute pas comme semblables (pour peu qu'ils se rencontrent et s'adressent la parole) et en tout cas probablement pas comme căldărari. Comme l'a montré P. Williams, «les classifications d'origine non-tsigane [...] ne donnent qu'une image schématique et, du point de vue sociologique, inexacte [des groupes tsiganes]. ${ }^{34}$ Les seuls termes correspondant à des entités socioculturelles réelles sont ceux mobilisés par les intéressés, au moins lorsqu'ils parlent de leurs semblables. Car en discourant sur les «autres Roms» (aver Roma), ils manipulent généralement les mêmes catégories vagues, et bien souvent fantasmées, que leurs voisins Gajé (cf. ci-dessous, l'exemple du village de M.).

Pour tenter de faire correspondre les diverses appellations avec des communautés réelles, il faudrait y appliquer le calque des entités locales et régionales qui composent la Roumanie d'aujourd'hui. Car la féodalité, persistante jusqu'au milieu du XIX ${ }^{\mathrm{e}}$ siècle, n'y a pas seulement favorisé une certaine spécialisation professionnelle des groupes locaux. Elle a surtout affirmé et pérennisé de fortes identités rurales de terroirs (le «pays », ţara en roumain), qu'il se soit agit de domaines seigneuriaux, ecclésiastiques ou bien de communautés de paysans libres. Contrairement à la France, où l'on veut aujourd'hui faire revivre ces terroirs après les voir vu disparaître ${ }^{35}$, ceux-ci ne sont jamais «morts »en Roumanie et marquent encore profondément les réalités contemporaines.

On retrouve ainsi à travers les régions une multitude de «pays » : țara Pădurenilor (« le pays des forestiers »), ţara Moţilor (« le pays des Moţi »), ţara Zarandului («le pays du Zarand »), ţara Haţegului («le pays du Hateg»), ţara Oaşului («pays d'Oaş»), Scaunul MureşMàrosszek («Siège de Mureş ») etc. Cette diversité de territoires issus de la féodalité, chacun porteur d'une identité locale particulière, explique la multitude des communautés roms roumaines, fruit de leur intégration locale multiséculaire, et permet ainsi de comprendre pourquoi celles-ci s'estiment différentes les unes des autres, puisqu'elles le sont, de fait.

Bien plutôt que membres d'une «minorité ethnique » nationale, les Roms définissent leur communauté en référence à une ville, un bourg ou un réseau de communes qu'ils présentent comme leur lieu d'origine et d'où proviennent tous leurs parents, au sens large (neam). Ceci est valable pour les Roms sédentaires autant que pour les groupes jugés « semi-nomades »: leur mobilité s'appuie sur un lieu d'origine et s'exerce dans un territoire donné.

\footnotetext{
${ }^{33}$ N.B. : Personne n'utilise jamais tous ces termes, mais seulement trois ou quatre d'entre eux, l'échantillon variant selon les régions. Par ailleurs, cette liste ne prétend pas à l'exhaustivité.

${ }^{34}$ Williams (1984), p. 89.

35 cf. E. Weber (1984); le titre original étant plus fidèle au sujet du livre: Peasants Into Frenchmen: The Modernization of Rural France, 1880-1914.
} 
Et c'est ainsi, ironie du sort, qu'une carte des terroirs folkloriques (les «pays »), produits de l'Âge féodal et, sous le régime communiste, symboles célébrés de l'autochtonie paysanne roumaine $^{36}$, permet de se faire une idée - au moins schématique - de la géographie des diverses communautés roms - cf. figure 6 .

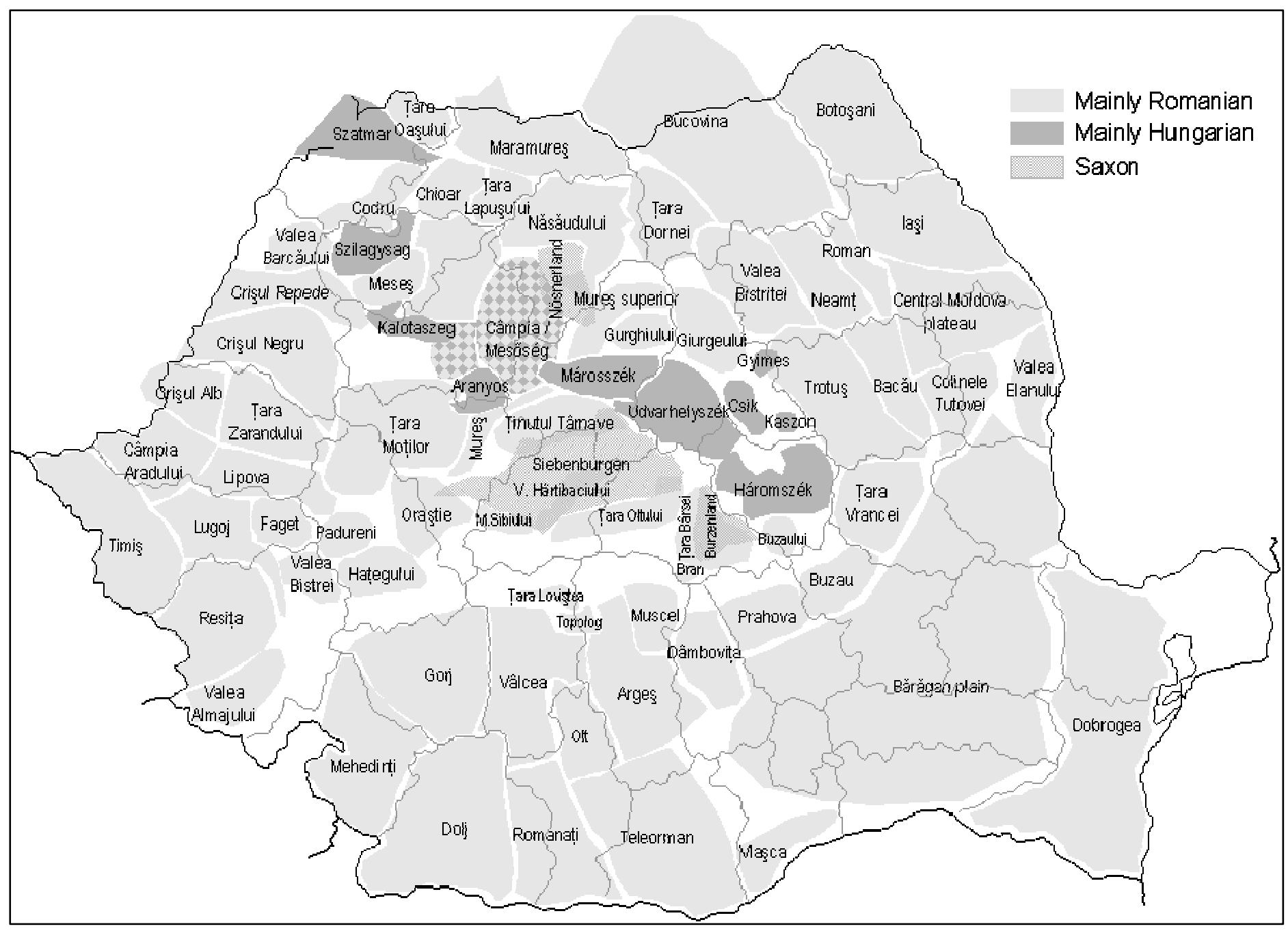

Figure 6. Zones ethnographiques de Roumanie d'après L. Mellish et N. Green - ( ) Eliznik2005

Certaines communautés sont importantes et très visibles, d'autres plus réduites et discrètes. Des groupes géographiquement proches le sont aussi culturellement, d'autres apparaissent objectivement très différents : différences de langue (roumaine ou hongroise, en plus du romanès le cas échéant) et différences d'accent dans celles-ci; différences vestimentaires ; différences de mode d'implantation locale (groupé ou dispersé) et donc de modes de relations avec le voisinage, lui-même divers (les Gajé); différences de rites matrimoniaux (fugue nuptiale, prix de la marié, dot...) ; différence de religion (orthodoxe, catholique, réformé, uniate, évangélique...), etc.

\footnotetext{
${ }^{36}$ Cf. Verdery (1995).
} 
La prononciation du terme Rom varie elle-même d'un groupe à l'autre ${ }^{37}$. Plus généralement, seuls les membres d'une même communauté parlent romanès de manière identique: les salutations, les façons de dire «oui », de souhaiter des bonnes choses (ou de s'insulter), de désigner nombre d'objets du quotidien etc., sont variables. Ces différences, parfois minimes lorsque les deux groupes proviennent de régions voisines, peuvent être rébarbatives, au point que les interlocuteurs préfèrent échanger en roumain. Mais encore faut-il, pour ce faire, qu'ils aient jugé opportun de s'adresser la parole... Dans tous les cas, seuls «nos Roms » (amare Roma, ţiganii noştri), ceux du vaste réseau de parenté originaires d'un même lieu, parlent romanès «parfaitement». Voilà ce que constatent toujours les Roms dans leurs interactions avec d'autres Tsiganes.

Il n'y a donc pas une seule et même manière «d'être Rom » (le romanès - la manière des Roms), seuls les observateurs extérieurs (savants ou non) cherchent, là encore, à établir une unité, quand les intéressés ne cessent de se distinguer les uns des autres et d'éprouver leurs différences au quotidien.

Et lorsque des Roms disent avec leurs voisins roumains et/ou hongrois du mal des ţigani, ils parlent des autres, ceux qui ne sont pas de leur communauté : ceux vus à la télévision, ceux d'une autre région; ces «autres » étant bien plus fantasmés qu'ils ne correspondent à des individus réels.

D'où la nécessité de bien distinguer les niveaux de discours :

- pour tous les habitants de Roumanie (Roms inclus), la figure symbolique du ţigan est problématique, pour des raisons liées à l'histoire du pays : elle a joué un rôle structurant dans l'élaboration du discours national au XIXe siècle ;

et dans le même temps :

- quasiment tous les habitants de Roumanie, plus encore en milieu rural, entretiennent des rapports quotidiens et étroits avec des Tsiganes (leurs amis, voisins, anciens collègues de classe, etc.), sans que cela ne pose problème à personne.

Il y a bien une distinction entre le «Nous » des Roms et le « Nous » des voisins avec lesquels ceux-ci vivent, les uns et les autres ne s'estimant pas en tout et pour tout semblables, mais les différences ne produisent pas nécessairement une violence, même symbolique, quotidienne. $A$ priori, les premiers ne marieront pas leurs fils avec les filles des seconds (bien qu'il y ait toujours eu des mariages mixtes) et chacun connaît les différences de l'autre : les Roms ne sont pas des Gajé, et réciproquement... Cela n'empêche pas de travailler ensemble, de trinquer au bar du village, de s'échanger des services ou de se prêter de l'argent, etc.

À noter qu'un Tsigane s'estime généralement plus proche d'un Roumain ou d'un Hongrois de la même région que de «son frère ethnique » d'une autre province, tel qu'on va le voir à travers l'exemple détaillé d'une situation locale.

\section{Le village de M. : un cas d'école?}

Sud de la Transylvanie: M., hameau d'un petit millier d'habitants, se trouve à une dizaine de kilomètres de Deva, ville de 70000 habitants $(89,2 \%$ de Roumains, $8,6 \%$ de Hongrois et 1,2\% de Romi, selon le recensement de 2002), préfecture du département de Hunedoara.

\footnotetext{
${ }^{37}$ D'où le caractère artificiel de toute graphie voulant correspondre à une prononciation unique (cf. les débats sur rom ou rrom).
} 
Le village est séparé en deux quartiers principaux par la route nationale, très fréquentée, doublée d'une voie ferrée. D'une part le sat («village») proprement dit, de l'autre un ensemble composé de deux entités : le târg («marché/foire ») et la colonia ${ }^{38}$.

Le sat est, comme son nom l'indique, la partie la plus ancienne du village. Aucune famille rom n'habite dans ce quartier, uniquement des Roumains installés pour la plupart de longue date. L'habitat y est composé de maisons avec cours, disséminées sur quatre rues, entre la voie ferrée et le fleuve Mureş. On trouve là trois églises (orthodoxe, réformée et grécocatholique - les deux dernières étant à l'abandon), l'école, la salle des fêtes municipale, le cabinet de consultation du docteur (doctor de familie) et un bar $^{39}$. Bien que plus étendu et regroupant une bonne partie des institutions locales, le quartier sat est démographiquement moins important que l'ensemble târg-colonia.

Le târg correspond à une foire agricole se tenant chaque premier samedi du mois. La route menant de la voie nationale au champ de foire (cf. plan ci-après : entre les points A et B strada târgului) est bordée de maisons rurales traditionnelles, avec cour fermée, et de quelques demeures récentes. Les habitants de cette rue longue de 500 mètres sont du même type que ceux du sat: on y trouve essentiellement des familles roumaines du cru, et quelques maisons hongroises.

C'est en bifurquant au bout de cette route (B), au-delà du champ de foire, que l'on arrive à la colonia proprement dite (point $\mathrm{C}$ ). À flanc de colline, cette dernière se compose d'un ensemble de trois immeubles de deux étages (les blocuri) et de deux longues baraques de plain-pied (barăci). Le tout a été construit à la fin des années 1960, lors de l'édification d'une centrale thermoélectrique sur le territoire de la commune. La colonia constituait le logement des ouvriers du chantier.

Aujourd'hui encore, une bonne partie des habitants de M., du sat comme de la partie târgcolonia, travaillent à la centrale. L'agriculture, plus ou moins développée selon les possessions de chacun, demeure néanmoins très présente au quotidien: micro-culture légumière (tomates, pommes de terre, oignons, poivrons...) et maïs pour les animaux (poules, vaches, cochons...). À la belle saison, les troupeaux d'oies occupent la chaussée et ses fossés. La haute silhouette des tours d'évacuation de la centrale thermoélectrique complète ce paysage industriel et campagnard.

À Deva et dans les environs, la colonia est regardée comme un lieu d'habitation de ţigani. Les chauffeurs de taxi font une moue dubitative lorsqu'un étranger demande à y être conduit.... À y regarder de plus près, les choses apparaissent néanmoins plus complexes : les Tsiganes sont loin d'être les seuls habitants de la colonia, et ceux y demeurant ne forment pas une communauté unique.

Plutôt qu'une appartenance « ethnique », ce qu'ont en commun les habitants de la colonia est d'être arrivés à $M$. au cours des quarante dernières années. La plupart des familles proviennent du centre de la Transylvanie, de villages magyars (en particulier du département de Mureş). Recrutées à domicile par les autorités, elles s'installèrent ici lors de l'ouverture du chantier de la centrale. Lorsque cette dernière fut terminée, la majorité des personnes resta sur place et s'y engagea, selon ses qualifications. Depuis maintenant une quinzaine d'années, la centrale n'embauche plus, bien au contraire. Les jeunes doivent chercher du travail ailleurs...

Contrairement aux autres habitants du village, de la partie sat mais également ceux riverains de la strada târgului, les résidents de la colonia ne possèdent pas de terres à cultiver, uniquement des jardins familiaux de taille modeste où poussent quelques tomates et couine

\footnotetext{
${ }^{38}$ D'autres habitations sont disséminées le long de la route, vers Deva, notamment de nouvelles maisons édifiées au cours des dernières années.

${ }^{39}$ Un second bar se situe de l'autre côté de la voie ferrée, à proximité immédiate de la route nationale : il peut donc être considéré comme «neutre », n'appartenant ni au sat ni à l'ensemble târg-colonia. Il est indistinctement fréquenté par les habitants des deux quartiers.
} 
éventuellement un cochon. Certains sont propriétaires de leur logement (dans les immeubles notamment - blocuri), les autres louent leur habitation à la centrale (celle-ci demeure propriétaire des baraques).

Les ţigani ne sont donc pas seuls dans la colonia. Y cohabitent des familles roms, hongroises, roumaines... et mixtes (rom-hongroise, roumaine-hongroise, rom-roumaine). Vivant ensemble depuis maintenant quarante ans, les uns et les autres entretiennent des relations de voisinage, d'amitié (et d'inimitié) ordinaires. Au quotidien, les «frontières ethniques » ne semblent ainsi pas décisives dans les interactions locales. Elles ne sont pas oubliées, mais ne conditionnent pas les relations interindividuelles. Autour d'un verre, les uns et les autres (Tsiganes, Roumains ou Hongrois) tombent bien plus souvent d'accord pour tracer une frontière commune entre eux-mêmes, les «locaux », et divers Autres (habitants de Bucarest, Moldaves, hommes politiques corrompus, fonctionnaires, étrangers, etc.), plutôt qu'ils ne s'efforcent de ségréguer les rapports sociaux du village. Cela serait d'autant plus compliqué à mettre en œuvre que tout un chacun sait d'expérience que les Tsiganes de M. n'appartiennent pas à un seul et même groupe. Une fois encore, une perspective locale met à mal les catégorisations univoques.

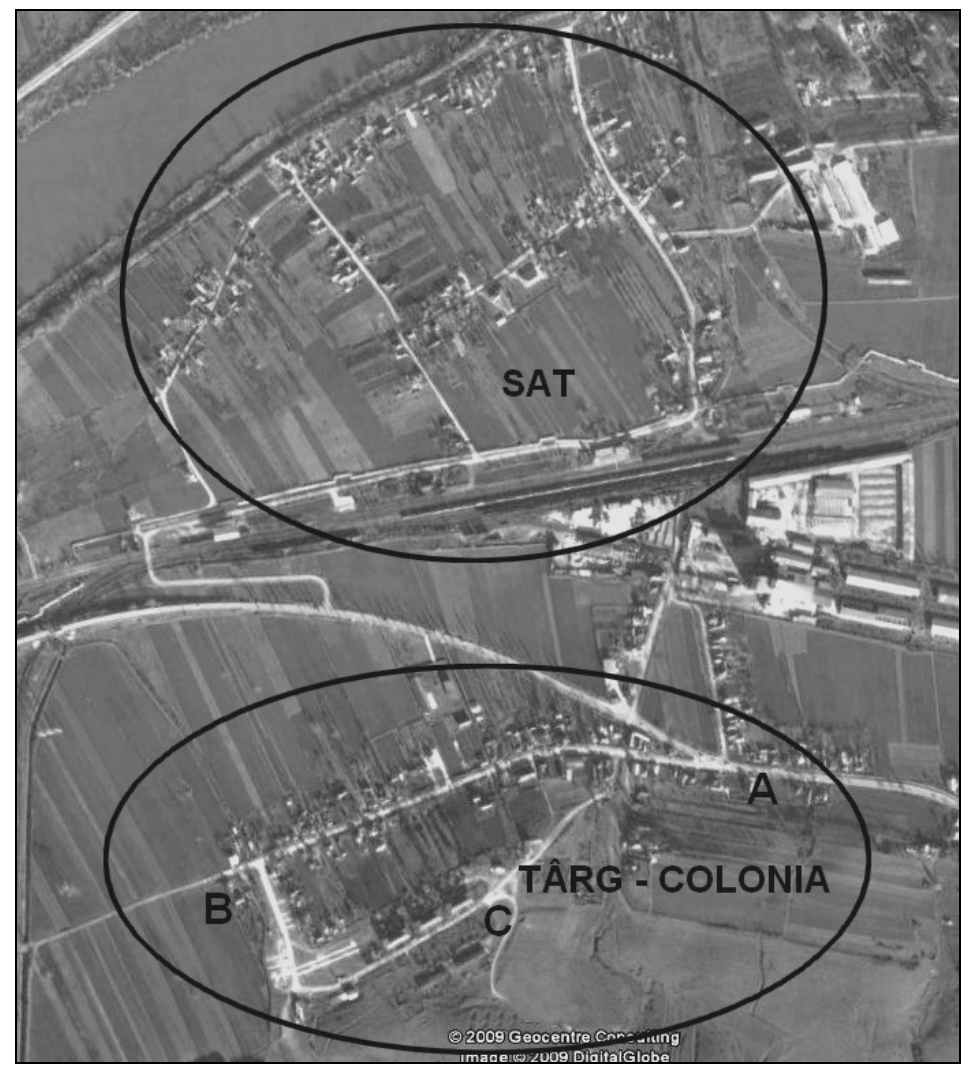

Figure 7. Le village de M.

Détaillons donc la présence tsigane à M. :

- Les plus nombreux, une douzaine de familles apparentées, soit une cinquantaine de personnes, sont des «Tsiganes roumanisés » : ils ne parlent que roumain et, pour les plus âgés, le hongrois (leurs vieux étant originaires des environs de la ville de TârguMures). Hormis un teint de peau généralement plus sombre, rien ne les distingue extérieurement de leurs voisins non-Tsiganes : ni la langue, ni les vêtements, ni les occupations professionnelles, etc. Certains vivent dans les baraques, d'autres dans les 
immeubles où ils sont propriétaires. On trouve un chauffeur de poids lourds, des engagés à la centrale, des artisans dans le bâtiment, des salariés dans des petites firmes locales, des émigrés en France ou Belgique, des inactifs ou chômeurs... Ils sont dits et se disent ţigani, mais ne mobilisent aucun ethnonyme spécifique - tandis que d'autres Roms les désignent comme rumunguri ou kaştale ${ }^{40}$. Depuis les années 1970, des mariages ont eu lieu entre des jeunes de ce réseau familial et des Hongrois ou Roumains de même niveau socio-économique, dans la commune ou dans les environs. Bien que les conditions de vie se soient améliorées au cours des dernières années et que toutes les familles de ce groupe ne soient pas pauvres, c'est parmi eux que l'on trouve les foyers les plus défavorisés de la colonia. Les habitants du village les regardent généralement comme les «cas sociaux» de la commune, fauteurs de scandaluri (beuveries, disputes, bagarres...). D’une manière générale, ces familles sont les destinataires des campagnes et/ou des projets de développement visant les Romi depuis 1990 : durant plusieurs années le monastère catholique hongrois de Deva assura sur place un jardin d'enfants (gradiniţa); diverses associations caritatives distribuèrent occasionnellement de l'aide aux familles; plusieurs jeunes participèrent à un projet de développement local mené par une association française (ouverture d'un atelier de menuiserie) qui prit fin en 1999. Enfin, depuis maintenant un an la mairie soutient une troupe de «danse folklorique tsigane»montée par une douzaine de jeunes issus de ces familles. Bien que ses membres ne soient pas locuteurs du romanès, la troupe fut nommée Kelen Amentza - «Dansez avec nous » en langue tsigane. La municipalité les sponsorisa pour l'achat de «costumes tsiganes traditionnels » et met à disposition la salle des fêtes lors des répétitions ;

- Un second ensemble familial est formé de Roms Gabori. Également originaires de la région de Târgu-Mureş mais d'un autre réseau de communes que les précédents, ils sont perçus comme des Tsiganes traditionnels : ils parlent romanès en sus du roumain et du hongrois, les femmes portent fichu coloré et longue jupe plissée, les hommes grandes moustaches, costume sombre et large chapeau de feutre noir. Ils sont par ailleurs connus comme ferblantiers-zingueurs et jouissent généralement d'une bonne réputation. Les familles gabori étaient plus nombreuses durant le chantier de la centrale, mais une bonne partie d'entre elles regagna sa région d'origine à la fin de celui-ci. Aujourd'hui, ces Roms comptent trois groupes domestiques à M. Le plus important, celui de Rupi (une douzaine de personnes), habite un peu à l'écart de la colonia, dans un bâtiment dont ils sont les seuls occupants, à hauteur du champ de foire. Rupi et les siens regardent les Tsiganes «du haut» (de la colonia stricto sensu, dont ils se distinguent géographiquement) comme des gens peu recommandables («sales, sans moralité, feignants...», soit tous les stéréotypes négatifs ordinairement associés aux ţigani). Ils les appellent rumunguri et s'évertuent à ne jamais mettre un pied chez eux. Leurs enfants ont en partie grandi avec ceux du haut (au moins à l'école), mais n'ont aujourd'hui gardé des contacts qu'avec deux ou trois «amis », faisant figure d'exceptions morales : «Eux ce sont des types bien ». D'une manière générale, la famille de Rupi entretient au quotidien davantage de rapports sociaux avec les Gajé du village (et, bien entendu, avec les autres familles gabori des communes alentours) qu'avec les Tsiganes de la colonia. Il n'y a là aucune "solidarité ethnique », bien au contraire ! Les membres de la famille sont connus dans le village pour être des "gens respectables », Rupi et son gendre entretenant dans tous les environs un vaste réseau de clients en couverture-zinguerie. Le frère de Rupi et ses enfants vivent quant à eux avec «ceux du haut», dans les baraques : s'habillant de

\footnotetext{
${ }^{40}$ Les deux termes sont généralement péjoratifs dans la bouche de ceux qui les emploient.
} 
manière moins «traditionnelle » que leurs oncle et cousins, ils se situent à mi-chemin entre Gabori et rumunguri et, dans l'ensemble, ne bénéficient pas de la bonne insertion locale dont jouit la famille de Rupi ;

Hormis ces deux principaux groupes familiaux, on trouve d'autres Tsiganes dans le quartier strada târgului-colonia. :

- Zoli et sa famille, originaires de Cluj, vivent à proximité des immeubles de la colonia, dans une maison indépendante. Issu d'une communauté de lăutari (musiciens), locuteur du romanès, le chef de famille travaille à son compte dans la maçonnerie, avec un certain succès. Il lui arrive occasionnellement de sortir son violon, sans toutefois en tirer de revenus ;

- La famille de Ioan est arrivée plus récemment dans le village. Madame est une Romni originaire du sud du pays, monsieur est Roumain (ou «Tsigane roumanisé »?) de Transylvanie. Elle parle quelques mots de romanès, avec un accent septentrional, mais s'habille sans signe distinctif, hormis un fichu... celui-ci n'est cependant pas l'apanage des «Roms traditionnels » : les paysannes le portent également. Le couple a ouvert un bar-épicerie peu de temps après son arrivée. Celui-ci, positionné près du champ de foire, est assez fréquenté, aussi bien par les Gajé de la strada târgului que par les habitants de la colonia. Leurs filles et beaux-fils sont émigrés en France ;

- Dans la strada târgului elle-même on trouve une famille dont la «tsiganité » peut être discutée : celle de Sandu. Selon les interlocuteurs et le contexte, Sandu et sa famille peuvent se dire ou être dits ţigani. Absolument rien ne les distingue pourtant du voisinage: ils ne sont que roumanophones, vivent dans une maison avec cours identique à celles des voisins, entretiennent avec ces derniers des relations parfaitement normales, possèdent un tracteur et quelques vaches, cultivent leur terre, ont marié leurs fils à de jeunes Roumaines des environs... Aux yeux des Gabori du village, ce ne sont même pas des rumunguri (la preuve : ils leur achètent du lait!), même s'ils évoquent parfois leur lointaine origine tsigane...

- Depuis maintenant une dizaine d'années, une famille de Roms cortorari vient monter sa tente à proximité du champ de foire. Originaires de Strehaia (ville du département de Mehedinţi, en Olténie) où ils possèdent une maison, ce sont des « chaudronniers », réparateurs d'alambics en cuivre. Ils s'installent ici quelques mois (généralement d'avril à septembre) afin de proposer leurs services dans les communes environnantes mais également pour collecter les métaux non ferreux. Tout comme les Gabori, ils sont perçus comme des «Roms traditionnels » (usage de la langue romanès, vêtements traditionnels, métier traditionnel, pratique du «semi-nomadisme»...). Du fait d'un voisinage maintenant régulier, la famille de Rupi et ces Roms ont établi des relations : en dépit des différences entre leur romanès respectif ${ }^{41}$, ils discutent ensemble régulièrement et s'échangent de menus services. Les uns et les autres ne s'identifient cependant pas comme membres d'une seule et même «nation », loin de là ${ }^{42}$. Ce sont des amis et voisins occasionnels, en aucun cas des semblables avec lesquels des unions matrimoniales seraient envisageables. Pour les rumunguri de la colonia, se

\footnotetext{
${ }^{41}$ Les différences dialectales sont tout autant liées à l'accent (hongrois pour les uns, olténien pour les autres) qu'au vocabulaire et à certaines tournures de phrase. L'intercompréhension demeure toutefois quasi-totale lorsque chacun y met du sien. De même, le «costume traditionnel » des uns et des autres diffère de diverses manières : taille des chapeaux, motifs, couleurs et plissage des jupes, port du fichu, tressage des cheveux, etc. Bref, les intéressés ne peuvent en aucun cas se méprendre quant à leur appartenance à deux communautés distinctes. Les Gajé qui les connaissent non plus.

${ }^{42}$ Pour les Gabori, ces Roms sont des ţerhari, traduction littérale de cortorari (ţerha $=$ «tente » en romanès). À noter que les Tsiganes de la colonia désignent eux-mêmes souvent les Gabori comme étant des cortorari... Les uns et les autres manipulent ainsi les mêmes catégories, mais n'y font pas entrer les mêmes entités.
} 
présentant comme «Tsiganes modernisés », ces cortorari sont l'archétype même des ţigani «arriérés » (înapoiaţi), «non-civilisés », perçus comme totalement illettrés et mariant leurs enfants en bas âge. Il leur arrive toutefois de faire affaire avec eux, en leur revendant quelques kilos de cuivre ou d'aluminium récupérés çà et là.

La présence tsigane apparaît ainsi diversifiée à M., comme dans bien d'autres communes transylvaines. Cette diversité est inconnue de ceux n'y ayant jamais mis un pied et regardant le village comme une colonia de ţigani, repère de délinquants en puissance ou, selon une autre perspective, lieu déshérité à secourir. Mais de la même manière, les villageois de $\mathrm{M}$. et de la région (Tsiganes, Roumains et Hongrois confondus) regarde souvent les habitants de Moldavie (Tsiganes ou Roumains) comme indistinctement «sous-développés » et «arriérés ». Les stéréotypes, quels qu'ils soient, demeurent puissants, pour ne pas dire inébranlables, tant que les discours se veulent génériques...

Lorsqu'on les écoute parler des ţigani (c'est-à-dire des autres qu'eux-mêmes), tous les Tsiganes de M. valident pleinement les préjugés courants en Roumanie, de diverses manières selon leur appartenance communautaire : les uns déprécient et/ou plaignent les rumunguri ou kaştale, les autres méprisent les cortorari etc. Seul l'observateur extérieur, pour lequel les Roms doivent former une entité signifiante sui generis, peut déplorer et/ou ne pas comprendre une telle situation. En réalité, le jeu des stéréotypes permet à ceux qui, dans un contexte particulier, les mobilisent ensemble (ici Roms et non-Roms) d'affirmer leur identité et, ce faisant, de nouer des relations intimes.

Par définition, les clichés n'opèrent pas localement, en tout cas pas de manière décisive : il n'est en effet plus question de catégories mais de personnes et de familles uniques et particulières. Les différences socioculturelles n'en sont pas pour autant niées ou effacées par les uns et les autres, mais elles n'apparaissent pas problématiques en elles-mêmes.

Comme ailleurs, c'est hors du champ local que se manifeste le plus fréquemment les discriminations en Roumanie, dans le cadre des relations impersonnelles de la vie « moderne » : au comptoir d'une banque, à l'hôpital, devant la justice (qu'on y soit victime ou accusé)... Bien heureusement, nombre de Roms connaissent personnellement des médecins ou avocats aux yeux desquels ils ne sont pas un ţigan mais $\mathrm{X}$ ou $\mathrm{Y}$.

L'anti-tsiganisme comme produit de la modernité, voilà une piste de recherches que l'exemple roumain, entre autres, invite à creuser plus avant.

\section{Conclusion}

On l'aura constaté, cet article invitait davantage à questionner la perspective à adopter lors de l'étude des réalités roms roumaines, plutôt qu'il n'a souhaité apporter des réponses définitives à des questions précises.

«Les Roms » sont-ils discriminés ? «Les Roms» sont-ils pauvres et marginaux ? «Les Roms » ont-ils honte de leur «appartenance ethnique »? Autant de questions auxquelles on sait désormais qu'il est impossible d'apporter des réponses globales et univoques. Car «les Roms », en tant que catégorie générique, ne forment ni un groupe social, ni une entité culturelle. Sans aller jusqu'au détail des situations personnelles et familiales, c'est au niveau des communautés locales, de quelques centaines ou milliers de familles selon les cas, que l'on peut saisir des réalités socioculturelles riches et variées, tout autant que des degrés divers d'intégration dans l'environnement.

Oui, des communautés roms sont pauvres et marginales et nécessitent la mise en œuvre de programmes de soutien dans différents domaines. Pauvreté et marginalité n'en constituent pas pour autant l'alpha et l'oméga des réalités roms roumaines. 
Oui, des Roms sont régulièrement confrontés à des attitudes discriminantes, liées aux préjugés tenaces structurant le champ symbolique national roumain, stéréotypes qu'ils sont par ailleurs les premiers à valider lorsqu'il s'agit de parler des «autres Tsiganes». De ce point de vue, les Roms de Roumanie sont bel et bien des nationaux...

Oui, certains Roms préfèrent se déclarer Roumains ou Hongrois aux recensements, plutôt que țigani ou Romi. Mais les motivations sont variées, et parfois contradictoires. Elles ne correspondent pas nécessairement à une «honte » de l'appartenance communautaire, c'est même parfois tout l'inverse : la fierté de former avec ses semblables une entité particulière (rasa, neam, naţia ou autre, les termes sont variables selon les groupes) interdit de s'identifier aux ţigani des représentations populaires.

On le sait, les années 1990 ont connu une quinzaine d'affrontements entre Roumains et Roms dans diverses communes du pays (agressions, incendies de maisons et, dans certains cas, mort d'hommes). La problématique «ethnique »n'est toutefois pas au fondement de ces violences. Ce sont plutôt des conflits locaux de voisinage qui ont dégénérés en affrontements collectifs et se sont à cette occasion ethnicisés.

L'omniprésence du «problème tsigane» dans la presse roumaine post- $1989^{43}$ n'a bien entendu pas arrangé les choses, mais il faut davantage se pencher sur les raisons politiques et sociales de ces tensions et violences (paupérisation généralisée du pays depuis les années 1980, démantèlement du secteur économique public et, ce faisant, enrichissement rapide d'une classe d' " homme d'affaires » - héritiers directs des apparatchiks d'antan - reconvertis au libre-échange débridé, redistribution aléatoire de terres agricoles, règlements de compte vis-à-vis d'anciens notables locaux, etc.), plutôt que sur un clivage " ethnique », si l'on veut en saisir les tenants et aboutissants. De ce point de vue, continuer d'aborder les Roms comme une «population», quelle que soit la perspective adoptée (sociale, folklorique, judiciaire, humanitaire ou autre), n'est sans doute pas de nature à faciliter l'insertion locale des familles les moins favorisées.

Ni harmonie parfaite, ni violence quotidienne, la réalité sociale se situe localement, là où les gens vivent ensemble, depuis parfois fort longtemps, et non au niveau des grands discours catégoriels, réifiant les identités collectives et figeant les dynamiques sociales dans des schémas univoques.

\footnotetext{
${ }^{43}$ Omniprésence proportionnelle aux injonctions occidentales sur la «question rom », érigée en étendard des problèmes de ces pays « en transition ». Mais, de ce point de vue, les institutions nationales d'Europe de l'Ouest n'ont pas nécessairement de leçons à donner sur la «politique tsigane »...
} 


\section{Références citées}

- Achim, V., The Roma in Romanian History, Central Europe University Press, Budapest, 2004

- Castellan, G., Histoire des Balkans, XIV -XX siècles, Fayard, Paris, 1991

- Comunitatile de Romi din România - O hartă a saraciei comunitare prin sondajul PROROMI, A.N.R., 2005

- Courthiade, M., «Quelques repères psych(olog)iques dans l'histoire des Roms de l'Est », in Les Familles roms d'Europe de l'Est, Cahiers de l'IDEF, Alizé, 1993

- Courthiade, M., «De Stefan Răzvan à "Răzvan si Vidra" », in L'esclavage des Rroms, Études tsiganes $n^{\circ} 29$, Paris, 2007

- Djuvara, N., Le pays roumain entre orient et occident, POF, Aurillac, 1989

- Formoso, B., Tsiganes et Sédentaires, la reproduction culturelle d'une société, L'Harmattan, Paris, 1986

- Houliat, B., Tsiganes en Roumanie, éditions du Rouergue, Rodez, 1999

- Olivera, M., Romanès, ou l'intégration traditionnelle des Gabori de Transylvanie, thèse de doctorat en ethnologie, Université Paris-X, 2007

- Olivera, M., «De l'usage de l'autochtonie chez les Gabori, Roms de Transylvanie. Dis moi d'où tu es, je te dirai qui tu es. », in Radicamento et circolazione dei Rom d'Europa, DiPAV-Quaderni $n^{\circ} 24$, Franco-Angeli, Milan, 2009

- Painatescu, "Gypsies in Wallachia and Moldova: a chapter of economic history », in Journal of the Gypsy Lore Society, Third series, vol. 20, n² 2, Edinburgh, 1941

- Piasere, L., Mare Roma, Catégories humaines et structure sociale, Une contribution à l'ethnologie tsigane, thèse de doctorat publiée par Études et documents balkaniques et méditerranéens, vol. 8, Paris, 1985

- Piasere, L., I rom d'Europa, Una storia moderna, Editori Laterza, Roma, 2004

- Potra, G., Contribuţiuni la istoricul ţiganilor din Romania, Curtea Veche, Bucarest, 2001 (1939).

- Rey, V., éd., Atlas de la Roumanie, CNRS-Libergéo-La Documentation français, Paris, 2000

- Reyniers, A., «Quelques jalons pour comprendre l'économie tsigane », in Économie tsigane, Études tsiganes, vol. 12, Paris, 1998

- Recensamantul populatiei si locuintelor 2002, INSSE, Bucarest

- Stewart, M., «Fils du marché, les maquignons tsiganes et le modèle anthropologique », in Jeux, tours et manège, une ethnologie des Tsiganes, Études tsiganes, 2/1994, Paris, 1994

- Vaux de Foletier, F., «L'esclavage des Tsiganes dans les Principautés roumaines », in Études tsiganes, 1970/3

- Verdery, K., National ideology under socialism, Identity and cultural politics in Ceausescu's Romania, University of California Press, 1995

- Weber, E., La fin des terroirs, la modernisation de la France rurale, Fayard, Paris, 1984 
- Williams, P., Mariage tsigane, Une cérémonie de fiançailles chez les Rom de Paris, L'Harmattan-Selaf, Paris, 1984.

- Zamfir, E. et C., Tiganii : între ignorare şi îngrijorare, Editura alternativa, Bucarest, 1993 


\section{L'ambiguiité télévisuelle}

La saison 2007-2008 fut marquée par le succès de la telenovela «Inimă de tigan » («Cœur de tsigane ») sur la chaîne privée Acasă TV. Produit intégralement roumain, cette série en 159 épisodes met en scène des «Tsiganes traditionnels », sur fond d'amour impossible entre le héros (Tsigane) et sa dulcinée (Roumaine). Réel phénomène médiatique, le feuilleton rafla régulièrement les meilleurs scores d'audimat. Le succès populaire fut indéniable, quelle que soit l'«appartenance ethnique »des téléspectateurs, au point qu'une suite se trouve sur le point de sortir. L'imagerie relative aux Roms mobilisée est essentiellement exotique et folklorisante : les acteurs jouant les Tsiganes de la série parlent bien évidemment roumain, mais avec un fort accent du sud du pays (censément « authentique »); les costumes apparaissent (très) librement inspirés des «vêtements traditionnels » tsiganes; les références plus ou moins ésotériques à la «loi tsigane »sont régulières, etc.

À la même époque, une chaîne concurrente (Pro Tv pour ne pas la citer) tenta de surfer sur la même vague, tout en exploitant une autre thématique : celle de Tsiganes «modernisés » et magouilleurs, cohabitant avec des Roumains dans un appartement, avec en toile de fond le thème de l'émigration et divers trafics. La série tourna court devant son incapacité à lutter contre les froufrous, jupes colorées et autres feux de camp d'Inimă de ţigan.

Autre phénomène télévisuel récurrent ces dernières années : l'association de stars des manele avec de grandes figures de la musique folklorique lors des shows musicaux du samedi soir (voire même du nouvel an). Ainsi d'une soirée sur Antena 1, grande chaîne privée, ayant comme invité d'honneur le fameux manelist Adrian Copilulul Minune (récemment rebaptisé Adi de Vito) et ses «guest stars ». Ces dernières étaient à part égal des chanteurs (tsiganes) de manele et des représentants de la «muzică populară » (i.e. musique folklorique paysanne), globalement plus âgés que les premiers... Chantant ensemble ou séparément leurs tubes respectifs, les uns et les autres apparaissaient à l'écran, bras dessus, bras dessous, comme les meilleurs amis du monde, depuis son origine.

On ne peut soupçonner ces chaînes de télévision d'opérer ainsi par souci du «politiquement correct », mais bien plutôt de viser le succès d'audimat. Et cela semble être manifestement payant.

$\mathrm{Si}$, d'après tous les sondages d'opinion, les Roumains ne peuvent pas voir les Tsiganes en peinture (mais l'on a vu quelle valeur accorder aux discours génériques), ils les recherchent manifestement à la télévision... dans des rôles bien précis qui, quoique stéréotypés, ne sont pas nécessairement négatifs. Voilà une nouvelle ambiguïté à explorer, afin de mieux comprendre cette intimité paradoxale structurant la société roumaine contemporaine. 